Delft University of Technology

\title{
Relation Between Geometry and Wake Characteristics of a Supersonic Microramp
}

Tambe, S.S.; Schrijer, F.F.J.; van Oudheusden, B.W.

DOI

10.2514/1.J059868

Publication date

2021

Document Version

Final published version

Published in

AIAA Journal

\section{Citation (APA)}

Tambe, S. S., Schrijer, F. F. J., \& van Oudheusden, B. W. (2021). Relation Between Geometry and Wake Characteristics of a Supersonic Microramp. AIAA Journal, 59(11), 4501-4513.

https://doi.org/10.2514/1.J059868

\section{Important note}

To cite this publication, please use the final published version (if applicable).

Please check the document version above.

Other than for strictly personal use, it is not permitted to download, forward or distribute the text or part of it, without the consent of the author(s) and/or copyright holder(s), unless the work is under an open content license such as Creative Commons.

\section{Takedown policy}

Please contact us and provide details if you believe this document breaches copyrights.

We will remove access to the work immediately and investigate your claim. 
Green Open Access added to TU Delft Institutional Repository

'You share, we take care!' - Taverne project

https://www.openaccess.nl/en/you-share-we-take-care

Otherwise as indicated in the copyright section: the publisher is the copyright holder of this work and the author uses the Dutch legislation to make this work public. 


\title{
Relation Between Geometry and Wake Characteristics of a Supersonic Microramp
}

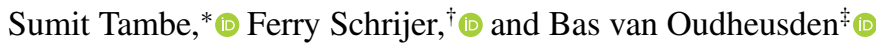 \\ Delft University of Technology, $2 \overline{6} 29 H S$ Delft, The Netherlands \\ https://doi.org/10.2514/1.J059868
}

\begin{abstract}
Microramp vortex generators are robust, reliable, and simple devices for passively controlling the boundary layer in several aerospace applications. Various past studies have investigated the effectiveness of microramps in controlling the flow separation induced by shock-wave/boundary-layer interactions. Building upon the past knowledge, this paper reports a systematic investigation of the relation between the microramp geometry and the downstream flow characteristics. A simplified flow model of the microramp wake is provided to explain and predict the influence of changing the geometry on the circulation of the primary vortex pair. The model also provides scaling relations for the evolution of the wake characteristics (that is, wake velocity, wake location, and added incompressible momentum), incorporating the effect of all geometry parameters. Extensive experimental data have been used to validate the model.
\end{abstract}

\section{Nomenclature}

\begin{tabular}{|c|c|c|}
\hline$b$ & $=$ & microramp span \\
\hline$C$ & $=$ & microramp edge \\
\hline E & $=$ & momentum \\
\hline$h$ & $=$ & microramp height \\
\hline$K_{\text {circ }}$ & $=$ & proportionality parameter relating to circulation \\
\hline$K_{R}$ & $=$ & model parameter relating to radius \\
\hline$K_{1}$ & $=$ & parameter relating to the circulation evolution \\
\hline$M$ & $=$ & Mach number \\
\hline$N$ & $=$ & ensemble size \\
\hline$P$ & $=$ & pressure \\
\hline$R$ & $=$ & vortex core radius \\
\hline$R e$ & $=$ & Reynolds number \\
\hline$U$ & $=$ & streamwise velocity \\
\hline$V$ & $=$ & wall-normal velocity \\
\hline$x, y, z$ & $=$ & coordinate axes \\
\hline$\Gamma$ & $=$ & circulation \\
\hline$\delta$ & $=$ & boundary-layer thickness \\
\hline$\epsilon$ & $=$ & uncertainty \\
\hline$\theta_{r}$ & $=$ & ramp angle \\
\hline$\theta_{s}$ & $=$ & half-span angle \\
\hline & $=$ & vorticity \\
\hline
\end{tabular}

\section{Subscripts}

$\begin{array}{ll}\text { added } & =\text { added by the microramp } \\ c & =\text { captured } \\ \mathrm{cc} & =\text { cross correlation } \\ i & =\text { incompressible } \\ u, v & =\text { relating to velocity components along } x \text { and } y \text { axes, } \\ & \text { respectively } \\ x, y, z & =\text { components along } x, y, \text { and } z \text { axes } \\ \tau & =\text { wall friction }\end{array}$

Received 8 June 2020; revision received 8 March 2021; accepted for publication 8 March 2021; published online 27 April 2021. Copyright (C) 2021 by the authors. Published by the American Institute of Aeronautics and Astronautics, Inc., with permission. All requests for copying and permission to reprint should be submitted to CCC at www.copyright.com; employ the eISSN 1533-385X to initiate your request. See also AIAA Rights and Permissions www.aiaa.org/randp.

*Ph.D. Candidate, Department of Aerodynamics, Wind Energy and Flight Performance and Propulsion, Faculty of Aerospace Engineering, Zuid Holland.

${ }^{\dagger}$ Assistant Professor, Department of Aerodynamics, Wind Energy and Flight Performance and Propulsion, Faculty of Aerospace Engineering, Zuid Holland.

\#Associate Professor, Department of Aerodynamics, Wind Energy and Flight Performance and Propulsion, Faculty of Aerospace Engineering, Zuid Holland.

$\begin{array}{lll}\omega & = & \text { relating to vorticity } \\ \infty & = & \text { relating to freestream } \\ \circ & = & \text { relating to stagnation condition }\end{array}$

\section{Introduction}

B OUNDARY-LAYER separation is a crucial problem that reduces the performance of aerospace systems, and therefore constrains their operational envelope. On transonic and supersonic vehicles, shock waves impose a sharp adverse pressure gradient on the boundary layer. The corresponding loss in momentum near the wall makes the boundary layer prone to separate. In transonic/supersonic engine inlets, the separated boundary-layer causes a total pressure loss that reduces the performance and stability margin of the engine. For supersonic and hypersonic vehicles, the shock-wave/boundary-layer interaction (SWBLI) can cause flow separation, which increases drag and reduces the vehicle performance. On a transonic wing, shock-induced boundary-layer separation can lead to violent buffeting, which puts limits on the flight envelope. Similar phenomena on compressor and fan blades may significantly reduce the engine efficiency. Therefore, the control of shock-wave/boundary-layer interaction is necessary to improve the performance of existing aerospace systems and expand the design space for future designs.

Traditionally, supersonic engine inlets feature a boundary-layer bleed (suction) system that removes the low-momentum boundary layer to avoid separation [1,2]. Alternatively, injection of high-momentum fluid into the boundary layer has also been shown to be effective in controlling the adverse effects of SWBLI [3]. However, these active flow control systems are often heavy and complex. A simple lightweight flow control technique is more desirable.

This makes the use of passive boundary-layer control techniques appealing, which use the momentum available in the flow to make the boundary layer more resistant to separation, either by promoting laminar to turbulent transition [4] or by redistributing the momentum by means of vortices. The latter is the specific working principle of vortex generators, which generate streamwise vortices to enhance mixing and transport the high-momentum fluid toward the wall. Consequently, the boundary layer has a fuller profile and can sustain higher adverse pressure gradients without separating.

In addition to strengthening the boundary layer, vortex generators also increase the drag. The wall-normal extent of their wake increases the boundary-layer thickness and may affect the outer flowfield [ $[5,6]$. The microvortex generator (MVG), submerged in the boundary layer, is aimed to reduce these adverse effects $[2,7,8]$. Among the different types of MVGs, vane geometries create the strongest vortex effect, but at the cost of higher drag and lower structural robustness [2]. Alternatively, microramps have lower drag, and their application is simple and more fail safe [9]. Therefore, microramps are usually considered 
as a potential candidate for passive boundary-layer control in actual applications.

Various past studies have assessed the effectiveness of microramps for flow control $[1,2,6,9-14]$. The flow structure of the microramp wake has been explored in detail both computationally and experimentally [5,15-24]. These studies have shown that microramps induce a streamwise counter-rotating vortex pair downstream. This vortex pair is surrounded by a train of hairpin-/ringlike vortices that are induced by the Kelvin-Helmholtz instability [18]. The momentum addition near the wall is primarily governed by the streamwise counter-rotating vortex pair (hereafter referred to as primary vortices), whereas the hairpin-/ringlike vortices only intermittently alter the effectiveness of the primary vortex pair $[2,18]$. The effects of Mach number, Reynolds number, and device height on the various characteristics of the microramp wake have been addressed [12,25]. Various microramp configurations have also been tested to mitigate the shock-induced separation [2]. The effect of different microramp sizes (i.e., different device heights relative to the oncoming boundary-layer thickness) on the wake properties has been investigated in the past $[25,26]$. These studies reported that the device height, regardless of the oncoming boundarylayer thickness, can be used as the primary length scale while scaling the microramp wake properties. However, in these studies, either the microramp geometry itself was kept constant or only very limited variations were considered. Therefore, although there is a basic understanding of the physical relation between the microramp geometry and the downstream flow characteristics $[17,27,28]$, a more systematic study is necessary to quantify these effects, which is the specific objective of the current investigation.

The impact of geometry is of evident relevance because an optimum microramp configuration will depend on the application, where the wake evolution has a significant effect. For example, the wall-normal extent of microramp wake may change the overall flowfield in an engine inlet; or, under size restrictions (intakes/compressor blades), a microramp that adds enough momentum near the wall within a short streamwise distance would be desirable. Therefore, understanding how changing the geometry affects the downstream evolution of the microramp wake is necessary to optimize the microramp flow control for a specific application.

This study furthermore aims to present a simplified physical flow model to describe how the geometry parameters of the microramp affect the wake flow characteristics. The central concept of the model is based on the captured momentum (i.e., the part of the oncoming flow momentum faced by the microramp) and how this is responsible for generating the streamwise vorticity. The model is further calibrated with data from a parametric experimental study, performed in a supersonic turbulent boundary (thickness of $\delta=6 \mathrm{~mm}$ ), by varying the half-span angle $\theta_{s}$ and ramp angle $\theta_{r}$ while keeping the device height of $h=4 \mathrm{~mm}$ constant. The wake characteristics (circulation, momentum, wake velocity and location, etc.) are quantified by measuring the flowfield using two-component (2C) and three-component particle image velocimetry (PIV). Schlieren imaging is used in addition to visualize the microramp wake evolution.

To predict the primary vortex circulation, the model is first calibrated with the experimental data from ramp angle sweep $\theta_{r}$ at a constant half-span angle $\theta_{s}$. The calibrated model is used to predict the data for the half-span angle sweep $\theta_{s}$ at a constant ramp angle $\theta_{r}$. The predictions are found to agree well with the measured circulations. Additionally, based on the concept of the captured boundary-layer momentum, appropriate scaling parameters for the evolutions of wake velocity, wake location, and added momentum are proposed. Discrepancies between model and experimental data are also identified and discussed.

\section{Experimental Setup}

\section{A. Flow Facility}

Experiments have been performed in supersonic wind tunnel ST-15 at the Aerospace Engineering Faculty of Delft University of Technology. The microramps were mounted on the test section floor for the PIV measurements and on the sidewalls for the top-view schlieren visualizations. The tunnel wall boundary layer is fully turbulent with a thickness of $\delta=6 \mathrm{~mm}$, as was measured by PIV. The incompressible friction velocity $u_{\tau}$ and skin-friction coefficient $C_{f}$ are obtained with the Clauser plot method [29] after applying the van Driest transformation [30] to the time-averaged boundary-layer profile [31]. The device height $(h=4 \mathrm{~mm})$ relative to the boundary-layer thickness is hence around $60 \%$. The freestream Mach number is $M_{\infty}=2$ in the investigation. Detailed data of the experimental conditions are shown in Table $\underline{1}$.

\section{B. Microramp Configurations}

The most common microramp configuration that has been considered by many researchers is the one resulting from the optimization study conducted by Anderson et al. [9], hence commonly referred to as the Anderson microramp. The flowfield behind the Anderson microramps has been explored in great detail in the literature $[9,16,18,25]$. Therefore, this configuration has been chosen as the baseline configuration for the present study, and other configurations are derived by varying geometrical parameter, one at a time. The microramp geometry is parameterized using the following parameters: height h, half-span angle $\theta_{s}$, and ramp angle $\theta_{r}$ (see Fig. 1). The trailing edge of the microramp is constrained to be perpendicular to the wall. Since the effect of varying the microramp height has already been widely explored in the literature [12], the focus is placed on the effect of the geometrical shape, and the microramp height is kept constant $(h=4 \mathrm{~mm})$ throughout the current study. The different test configurations investigated are obtained by varying $\theta_{s}$ (half-span sweep) and $\theta_{r}$ (ramp sweep), as documented in Table 2 .

\section{PIV Arrangement}

The flowfield in the symmetry plane of $z / h=0$ of the microramp has been investigated with two-component planar particle image velocimetry (2C-PIV). Three cameras, with a field of view of $10.17 \mathrm{~h} \times$ $7.7 h$ each, were used to measure the microramp wake from $x / h=6$ to 29 and $y / h=0$ to 5 . In addition, the flowfield in the crossflow plane is measured using three-component stereographic PIV at the streamwise locations of $x / h=5$ and $x / h=15$. The flow was seeded with diethyl-hexyl-sebacate tracer particles with a median diameter of $1 \mu \mathrm{m}$ [32] and a response time of approximately $2 \mu \mathrm{s}$ [33]. A doublecavity $\overline{\mathrm{Nd}}$ :YAG Spectra-Physics Quanta-Ray PIV-400 laser was used for the illumination. Specifications of the PIV setup are summarized in Table $\underline{3}$.

Table 1 Flow conditions and undisturbed boundary-layer properties

\begin{tabular}{lc}
\hline \hline Property & Value \\
\hline Freestream Mach number $M_{\infty}$ & 2.0 \\
Total pressure $P_{o}, \mathrm{~N} / \mathrm{m}^{2}$ & $3.15 \times 10^{5} \pm 0.03 \times 10^{5}$ \\
Freestream velocity $U_{\infty}, \mathrm{m} / \mathrm{s}$ & $487 \pm 7$ \\
Reynolds number based on height $R e_{h}$ & $1.85 \times 10^{5} \pm 0.08 \times 10^{5}$ \\
Friction velocity $u_{\tau}, \mathrm{m} / \mathrm{s}$ & $19.5 \pm 0.3$ \\
Skin-friction coefficient $C_{f}$ & $1.9 \times 10^{-3} \pm 0.1 \times 10^{-3}$ \\
Incompressible momentum thickness, mm & $0.52 \pm 0.02$ \\
Reynolds number based on momentum thickness & $2.4 \times 10^{4} \pm 0.1 \times 10^{4}$ \\
\hline \hline
\end{tabular}

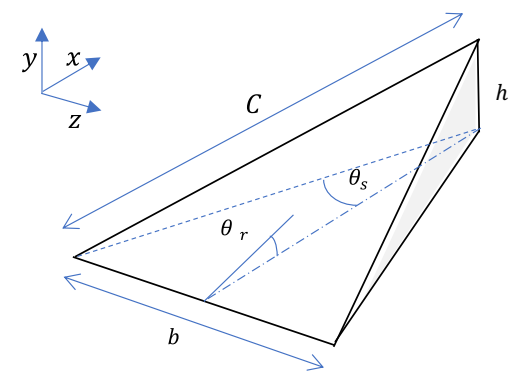

Fig. 1 Microramp geometry. 
Table 2 Configuration matrix

\begin{tabular}{lccccc}
\hline \hline Configuration & $\begin{array}{c}\text { Height, } \\
\mathrm{mm}\end{array}$ & $\begin{array}{c}\text { Half-span } \\
\text { angle } \theta_{s}\end{array}$ & $\begin{array}{c}\text { Ramp } \\
\text { angle } \theta_{r}\end{array}$ & $\begin{array}{c}\text { Width } \\
b, \mathrm{~mm}\end{array}$ & $\begin{array}{c}\text { Edge } \\
C, \mathrm{~mm}\end{array}$ \\
\hline MR24 (baseline) & 4 & 24 & 8.64 & 23.4 & 28.8 \\
MR20 & 4 & 20 & 8.64 & 19.1 & 28.0 \\
MR28 & 4 & 28 & 8.64 & 28.0 & 29.8 \\
MR36 & 4 & 36 & 8.64 & 38.2 & 32.5 \\
MR45 & 4 & 45 & 8.64 & 55.2 & 39.0 \\
MR4 & 4 & 24 & 4.3 & 47.4 & 58.2 \\
MR6 & 4 & 24 & 6.45 & 31.5 & 38.7 \\
MR11 & 4 & 24 & 11 & 18.3 & 22.5 \\
MR21 & 4 & 24 & 21.3 & 9.0 & 11.0 \\
\hline \hline
\end{tabular}

Table 3 Specification of the PIV setup

\begin{tabular}{|c|c|c|}
\hline & Planar PIV & Stereog raphic PIV \\
\hline Camera & $\begin{array}{l}\text { 3x LaVision } \\
\text { imager LX (2M) }\end{array}$ & $\begin{array}{c}\text { 2x LaVision } \\
\text { imager LX (2M) }\end{array}$ \\
\hline Camera resolution & $1624 \times 1236$ & $1624 \times 1236$ \\
\hline $\begin{array}{l}\text { Spatial resolution, } \\
\text { pixels/mm }\end{array}$ & 39.9 & 49.9 \\
\hline Pulse separation, $\mu \mathrm{s}$ & $1 \mu \mathrm{s}$ & $1 \mu \mathrm{s}$ \\
\hline Recording rate, $\mathrm{Hz}$ & 5.05 & 5.05 \\
\hline $\begin{array}{l}\text { Freestream particle } \\
\text { displacement, } \mathrm{mm}\end{array}$ & 0.5 & 0.5 \\
\hline Laser sheet orientation & Streamwise & Crossflow \\
\hline Laser sheet thickness, mm & $1.4-2$ & 2.5 \\
\hline
\end{tabular}

DaVis 8.1.2 software from LaVision has been used for data acquisition and processing. First, the particle images were preprocessed to minimize light reflections by subtracting the time minimum from the individual snapshots. The effects of inhomogeneous illumination are compensated for by dividing the snapshots by the average of the particle images. The vector calculation is carried out with a multipass approach. In the case of planar PIV, the window size is varied from $96 \times 96$ pixels (square, $50 \%$ overlap) to a final size of $32 \times 32$ pixels (elliptical $4: 1,75 \%$ overlap). In the case of stereographic PIV, the window size is varied from $64 \times 64$ pixels (square, $50 \%$ overlap) to a final size of $32 \times 32$ pixels (circular, $75 \%$ overlap). These settings result in a vector pitch of $0.2 \mathrm{~mm}$ for the 2C-PIV measurements and $0.16 \mathrm{~mm}$ for the stereographic PIV measurements. For each test configuration, a total of 400 PIV image pairs were recorded.

\section{Schlieren Imaging}

The schlieren images are acquired with a PCO Sensicam (1.3 megapixels). A spark lamp with an exposure time of $20 \mathrm{~ns}$ is used to illuminate the test section. A background image of an empty test section without the flow is subtracted from the dataset to eliminate the effect of imperfections in the optics. An average image is computed over an ensemble containing 300 images.

\section{E. Uncertainty Analysis}

The various types of uncertainties involved in the present measurements are summarized in Table $\underline{4}$, and the procedure to determine these uncertainties is outlined in Ref. [18]. The statistical-convergence uncertainties in the velocities $\left(\varepsilon_{u}\right.$ and $\overline{\varepsilon_{v}}$ ) are estimated using the rms

Table 4 Uncertainty analysis

\begin{tabular}{lc}
\hline \hline Uncertainty parameter & Value MR24 $(N=400)$ \\
\hline Statistical $\varepsilon_{u} / U_{\infty}$ & $<0.014$ \\
Statistical $\varepsilon_{v} / U_{\infty}$ & $<0.015$ \\
Cross correlation $\varepsilon_{\mathrm{cc}} / U_{\infty}$ & $<0.005$ \\
Spatial resolution $\Delta u / u_{o}$ & $<0.03$ \\
Vorticity $\varepsilon_{\omega} h / U_{\infty}$ & $<0.12$ \\
\hline \hline
\end{tabular}

of the velocity fluctuations and the ensemble size [34]. The velocity uncertainty due to the cross correlation is estimated using the crosscorrelation error ( 0.1 pixels), spatial resolution, and pulse separation [11]. The uncertainty due to the spatial resolution $\left(\Delta u / u_{o}\right.$, where $u_{o}$ is the actual flow velocity) is estimated using the interrogation window size with respect to the typical wavelengths in the flowfield [35]; in the present study, the latter relates to the wavelength of the hairpin- $/$ ringlike vortices in the instantaneous wake field of the microramp, which for the baseline model is around $1.5 \mathrm{~h}$ at $x / h=10$ [5]. The presented values apply to the baseline configuration. The uncertainty in the vorticity $\varepsilon_{\omega}$ is estimated using the vector spacing and the spatial velocity differences [18].

In addition to the uncertainties related to the measurement technique, practical uncertainties should be considered. Great care was taken to position the microramp at the required location by using a template; however, there may still be minor positioning errors that result in a small yaw angle. For the present experiments, it is estimated that this misalignment is less than $1 \mathrm{deg}$. The laser sheet used for the PIV measurements has a thickness between 1.4 and $2 \mathrm{~mm}$, and the measured flowfield is an average over the light sheet thickness.

\section{Flow Organization}

Figure 2 shows a conceptual sketch of the primary vortices around and downstream of the microramp in a time-averaged flowfield. The oncoming undisturbed boundary-layer flow undergoes compression when it encounters the leading edge of the microramp. The flow subsequently travels over the ramp surface and leaves at the microramp edge, which is the side edge labeled as $C$ in Fig. 1 . The flow on the microramp surface turns toward the edge before it separates [16]. Following the flow separation at the edge, the static pressure near the side of the microramp drops, leading toward the formation of the primary vortex. Also, part of the vorticity from the oncoming boundary layer is transferred to the primary vortex. The vortices from the two sides of the microramp approach each other just downstream of the trailing edge, where they form a counter-rotating vortex pair, creating the mutual upwash. Further downstream of the interaction zone, the primary vortex cores move away from each other as they start to entrain the outer high-momentum fluid. This leads to the increase in their size, and their vorticity starts to get diffused over a larger area. The mutual upwash also results in the liftup of the primary vortices. As the oncoming momentum is redistributed into the primary vortices, a deficit of streamwise momentum (wake) exists just downstream of the microramp (MR). During the primary vortex evolution, this wake also gets filled up by the entrained high-momentum flow. As they evolve downstream, the primary vortices bring the higher-momentum flow closer to the wall as well, which makes the boundary-layer profile fuller.

\section{Experimental Results}

To support this conceptual description, the results of the experimental parametric study are documented. First, the typical time-averaged wake topology is presented here, using the velocity fields obtained with PIV for the baseline configuration (MR24). Streamwise $U$ and wall-normal $V$ velocity contours in the symmetry plane are shown in Fig. 3. The momentum deficit region originates just downstream of the microramp, and it gets filled up when moving in the downstream

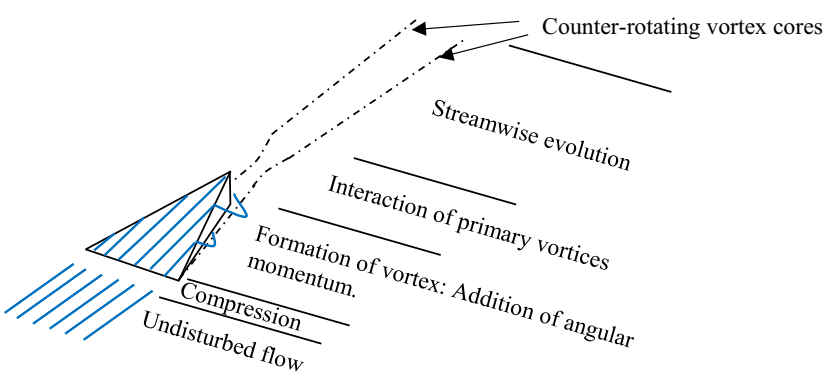

Fig. 2 Conceptual sketch of the evolution of the primary vortices around and downstream of the microramp. 


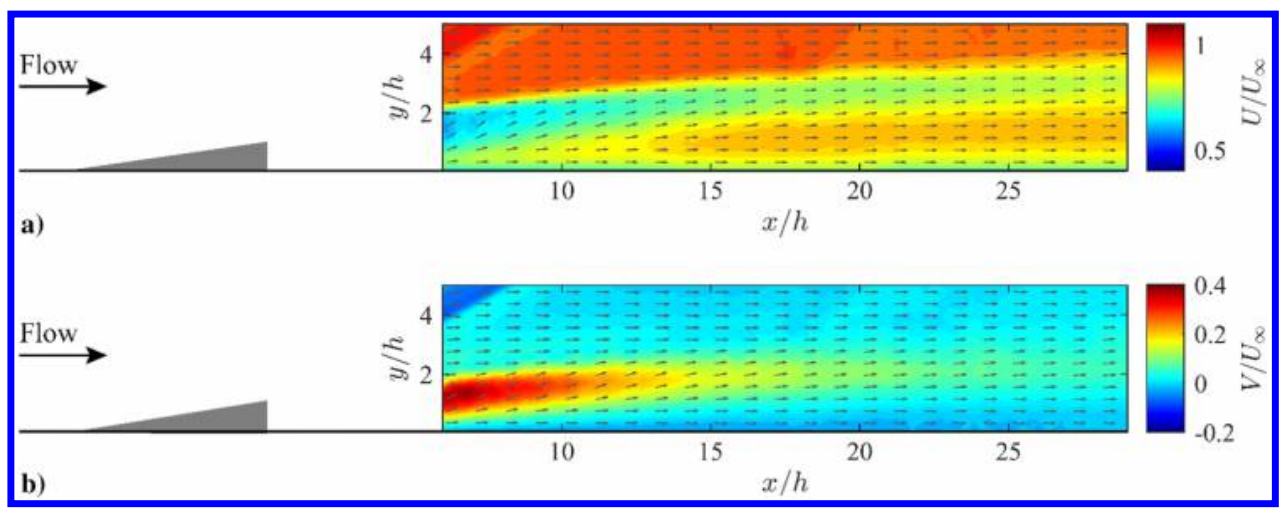

Fig. 3 Contours of a) streamwise velocity and b) wall-normal velocity in the symmetry plane of the microramp as measured with PIV (baseline configuration: MR24).

direction (see Fig. 3a). Here, the liftup of the wake can be observed clearly. Figure $3 \mathrm{~b}$ shows that the mutual upwash is stronger near the microramp and reduces further downstream. The near-wall streamwise momentum increases downstream (see Fig. 3a), and the momentum addition is apparent from slightly negative values of the wall-normal velocity component near the wall (see Fig. 3 b).

The spanwise extent of the wake is visualized using the crossflow PIV planes in Fig. 4, colored with contours of streamwise velocity. Here, the in-plane vectors clearly reveal the primary vortex pair, and the contours indicate the momentum addition near the wall. The highestmomentum addition near the wall is observed at locations away from the symmetry plane and approximately beneath the vortices; see Fig. 4a.
Further downstream (see Fig. 4b), the wake has been filled up while the vortices have decayed in strength.

The typical effect of changing half-span $\theta_{S}$ and ramp angle $\theta_{r}$ on the wake properties can be seen in the velocity profiles at the symmetry plane. Figure 5 shows the streamwise velocity profiles for different configurations at a fixed streamwise location $(x / h=10.7)$. Between the two inflection points, a minimum of the streamwise velocity is observed, which is referred to as the wake velocity (or maximum velocity deficit), as, for example, pointed out in Fig. 5 b for MR4; and its wall-normal location is referred to as the wake location. These two wake properties represent the evolution of the wake, and their scaling is discussed in Sec. VI.

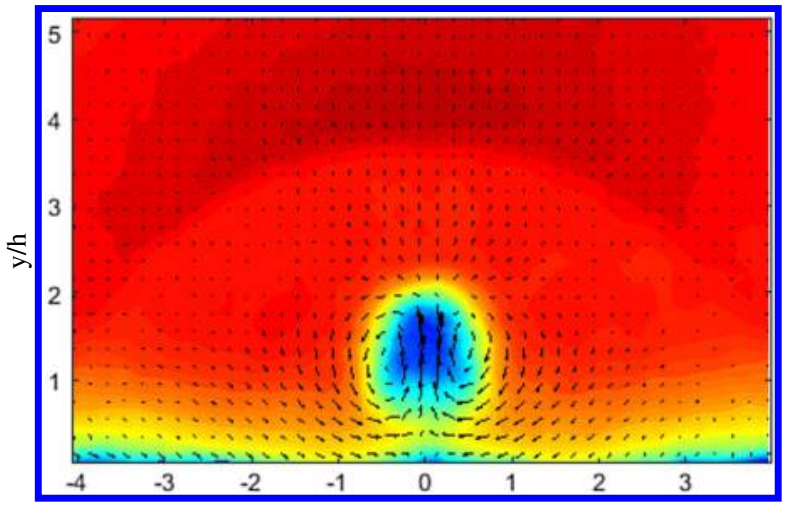

a)

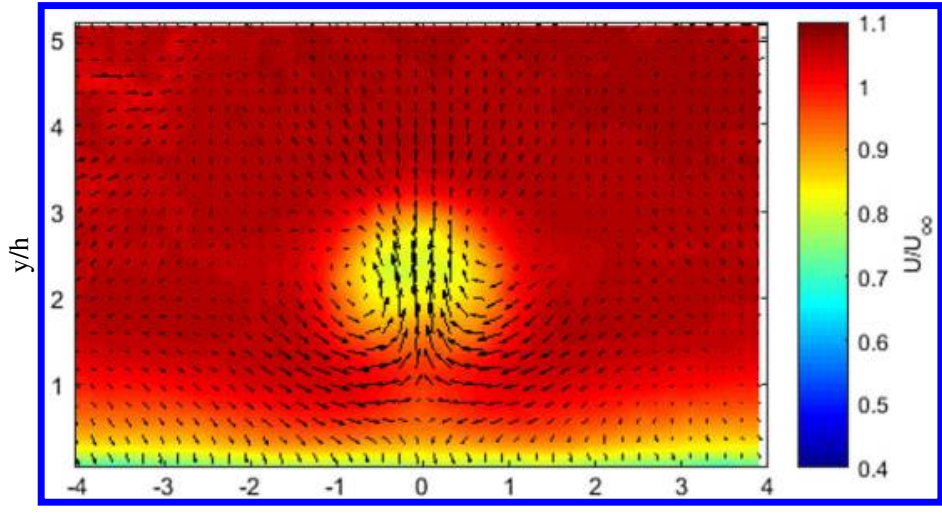

b)

Fig. 4 Flowfield in the crossflow planes with the contours of streamwise velocity at a) $x / h=5$ and b) $x / h=15$ (baseline configuration: MR24).

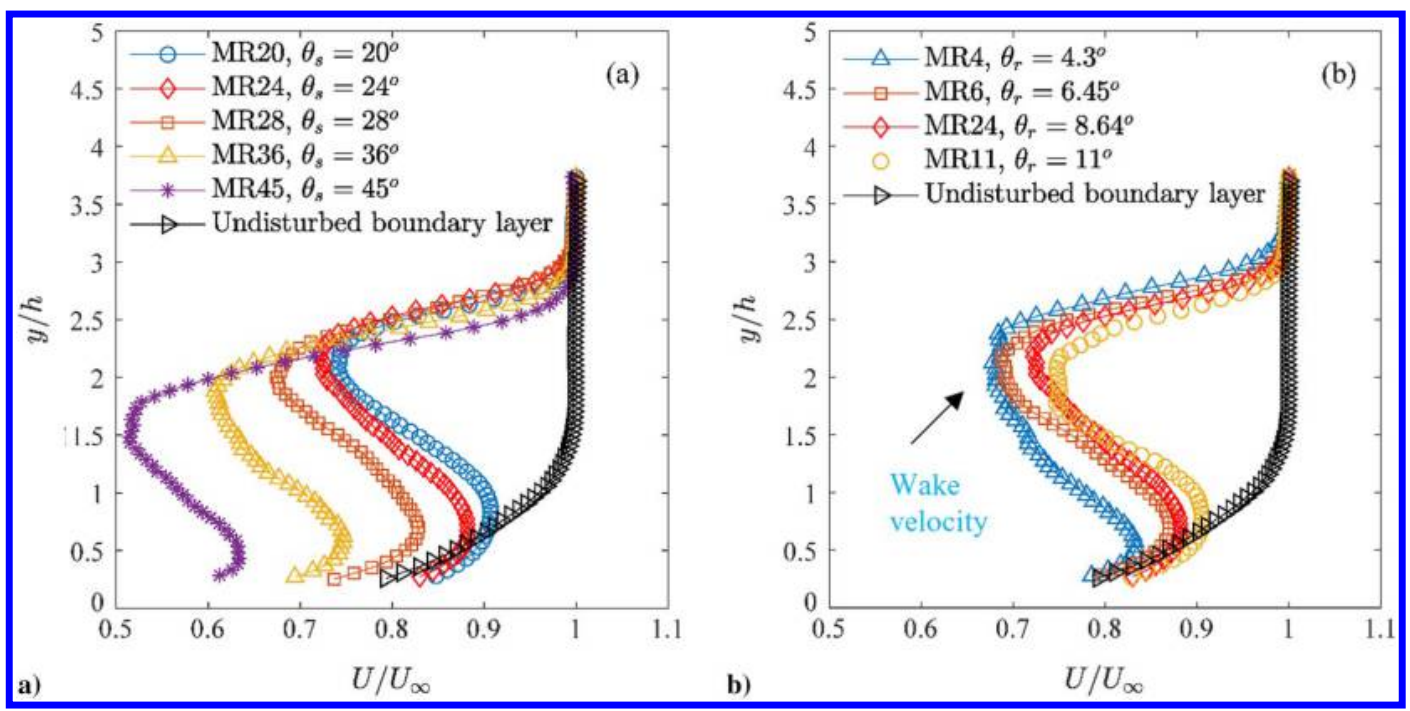

Fig. 5 Streamwise velocity profiles in the symmetry plane measured at $x / h=10.7$ : a) half-span angle sweep and b) ramp angle sweep. 
Streamwise velocity profiles (measured at $x / h=10.7$ ) in Fig. $\underline{5}$ show that the momentum addition phase of all the configurations is not yet complete; here, a clear momentum deficit is still present. However, the velocity profiles of some cases are already fuller near the wall than that of the undisturbed boundary-layer. These cases include microramps with $\theta_{s} \leq 24 \mathrm{deg}$ and $\theta_{r}>8.65 \mathrm{deg}$. This represents the momentum addition near the wall and will be further discussed in Sec. VI.D.

Figure 6 shows the wall-normal velocity profiles for the different configurations. For each case, the maximum wall-normal velocity occurs at the location of the strongest mutual upwash of the primary vortex cores. It is interesting to note that the ramp angle has a higher effect on the maximum upwash velocity than the halfspan angle. Comparing Figs. 5 and 6 shows that the wall-normal locations of maximum wake velocity and maximum upwash do not coincide, although their trend with respect to changes in geometry is similar.

Figure 7 shows the streamwise evolution of the spanwise core separation of the primary vortices for all the test cases obtained from PIV and ensemble-averaged schlieren images. The grayscale levels in schlieren images correspond to the spanwise (along the $z$ direction) density gradient in the flow. Moving away from the symmetry plane $(z=0)$, the grayscale sharply changes between bright and dark, indicating a reversal of the density gradient. This location is associated with the vortex core location (see figure 9.8 of the work of Giepman [5] for further details). At a constant microramp height, both the larger half-span angle and smaller ramp angle lead to the larger width of the microramp. Consequently, the vortex core separation is wider for the larger width. The core separation in the case of ramp angle sweep can be related to the increased core size and a stronger vortex. This is also evident from the corresponding increase in the mutual upwash (Fig. 6b). Near the microramp trailing edge $(x / h=0)$, the core separation is more sensitive to the changes in the half-span angle than the changes in the ramp angle.

Figure 7 also shows the core separations obtained from the stereographic PIV in the crossflow plane at $x / h=5$. Here, the local minimum of the crossflow velocity magnitude $\left(\sqrt{V^{2}+W^{2}}\right.$, where $V$ and $W$ are wall-normal and spanwise velocity components, respectively) is associated to the vortex core location, with the uncertainty of the order of one vector pitch: $\pm 0.04 \mathrm{~h}$. The comparison shows slight differences between the core separation obtained by schlieren images and PIV; however, the trends with respect to the half-span angle and ramp angle remain unchanged.

Figure 8 shows the top view of the microramp wake observed using the ensemble-averaged schlieren images, marked by the red lines. With increasing span angle, the separation (spanwise distance) between the vortex cores is larger, and they interact at a further downstream location; this is also evident by comparing crossflow fields at $x / h=$ 5 in Figs. 4a, 9a, and 9b. For large half-span angles, the streamwise momentum deficit region is closer to the wall due to the delayed interaction of the farther separated primary vortices. Although lowering the ramp angle increases the microramp width (similar to increasing half-span angles; see Table 2), it does not show a drastic increase in the vortex core separation (see Figs. $\underline{4 a}, \underline{10 a}$, and $\underline{10 \mathrm{~b}}$ ); the near-wall

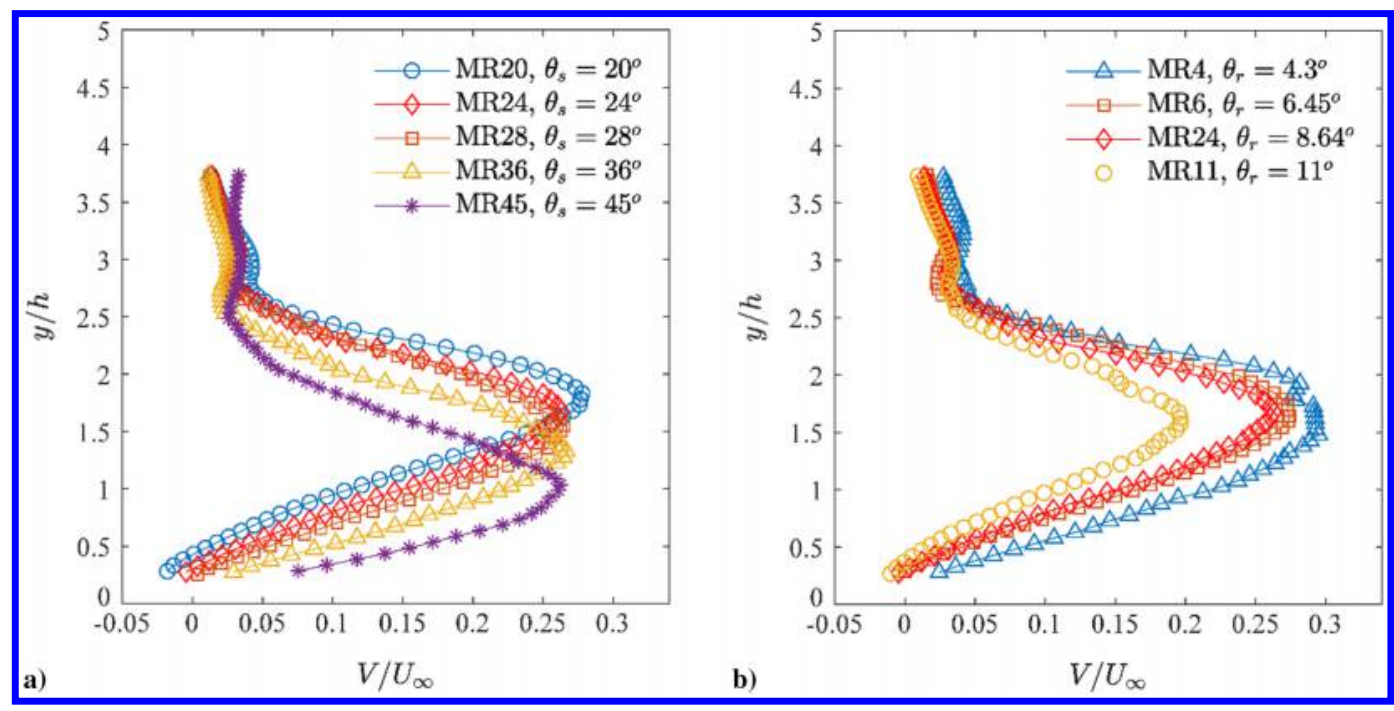

Fig. 6 Wall-normal velocity profiles in the symmetry plane measured at $x / h=10.7$ : a) half-span angle sweep and b) ramp angle sweep.
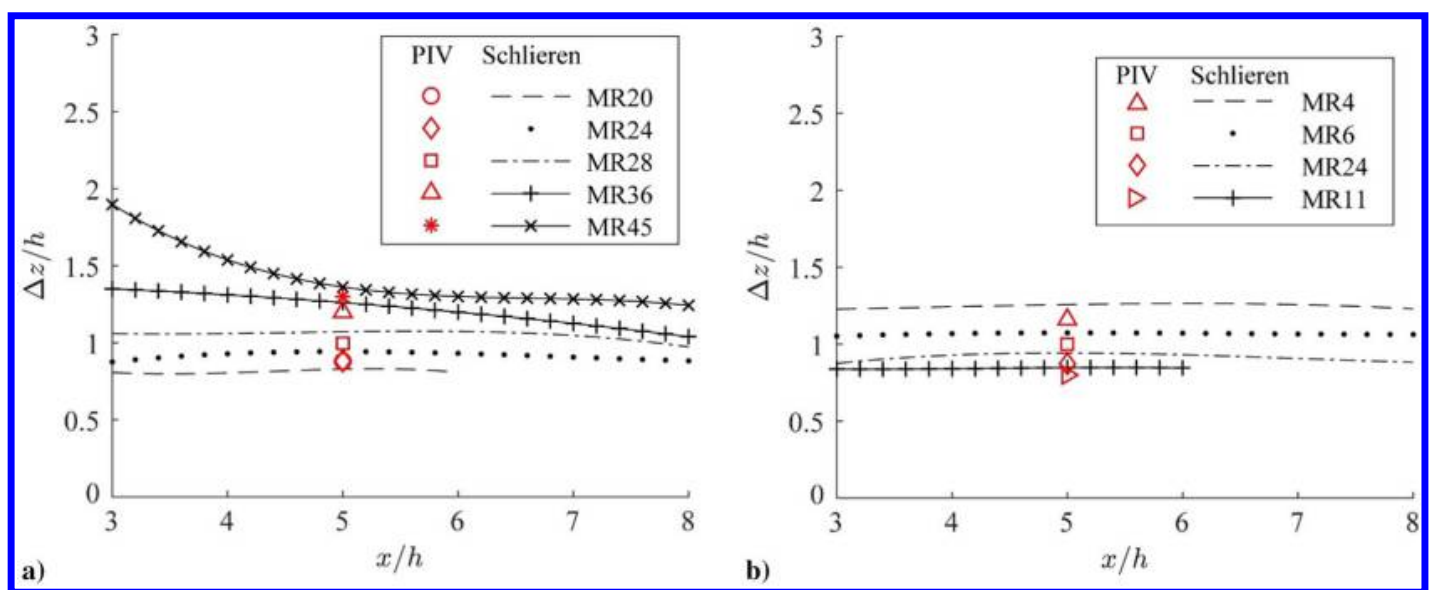

Fig. 7 Streamwise evolution of the primary vortex core separation: a) half-span angle sweep and b) ramp angle sweep. Core separations measured at $x / h=5$ using stereographic PIV are also shown for comparison. 

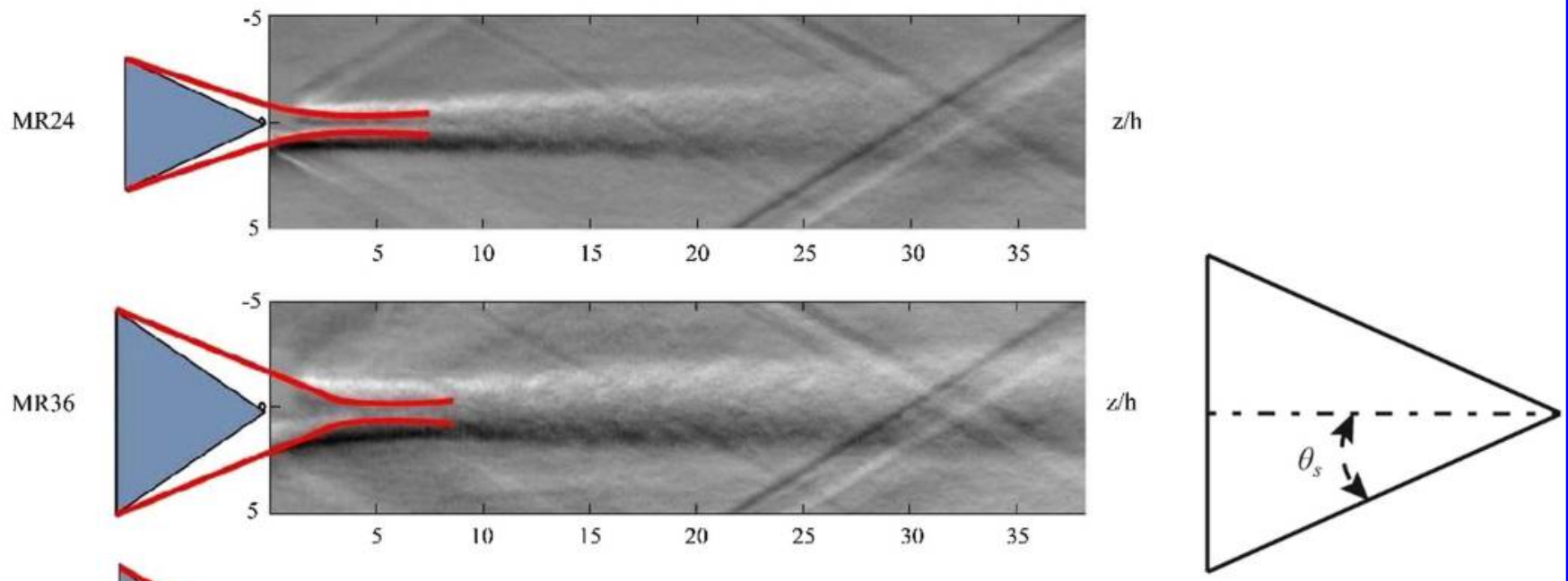

b)

MR45

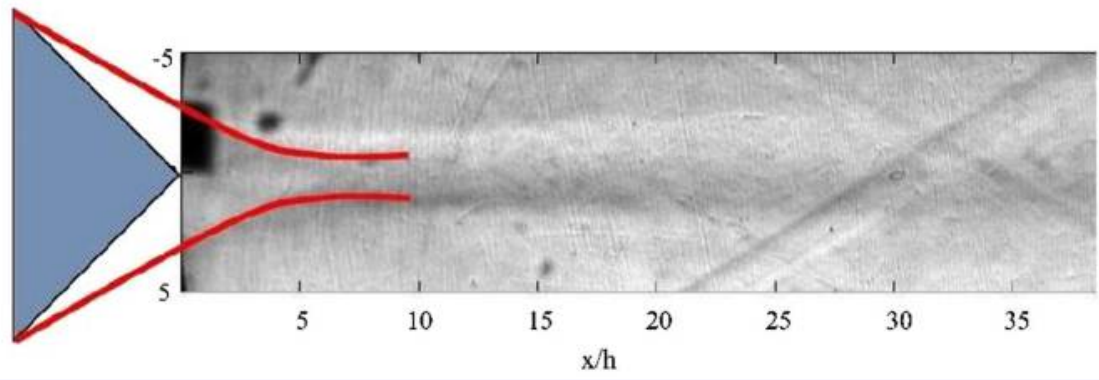

/h

a)

Fig. 8 Representations of a) effect of larger half-span angle on the core separation observed using the ensemble-averaged schlieren images (top view), and b) half-span angle $\theta_{s}$.
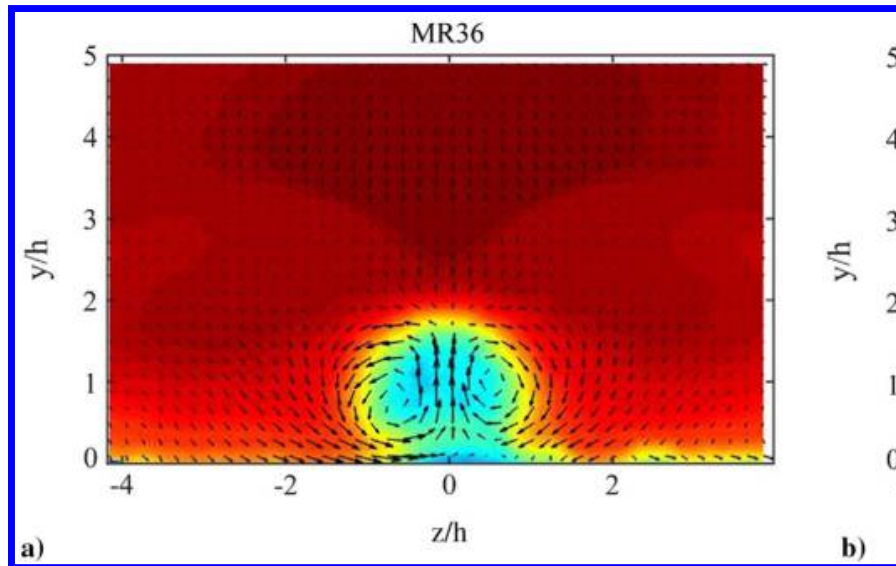

MR45

Fig. 9 Effect of large half-span angles on the crossflow field at $x / h=5$ with the contours of streamwise velocity: a) MR36 and b) MR45.

streamwise momentum deficit increases only slightly for MR4, which corresponds to the lowest investigated ramp angle.

\section{Flow Model}

Using information from the literature and the present experiments, a simplified physical model of the flowfield has been constructed to explain the effect of the microramp geometry on the flow properties of the wake. Furthermore, using this model, a new scaling is proposed (and validated) that takes into account the effect of the microramp geometry on the wake flowfield.

\section{A. Central Concept}

The microramp is a passive device that partly redistributes the momentum of the oncoming flow in order to create streamwise vorticity in its wake. The redistribution simultaneously results in a region of streamwise momentum deficit (the wake). The primary vortices are responsible for the transverse flow mixing as well as the subsequent filling of the wake, whereas the drag of the device mainly depends on the momentum deficit region. The strength of the primary vortices and the flow characteristics of the momentum deficit region depend on the amount of flow momentum that encounters the microramp. This momentum that crosses over the microramp leading edge is denoted here as the "captured momentum." Figure 11 illustrates the relation between the captured momentum and the microramp geometry. Considering one half of the microramp geometry, this momentum is then partially transferred into a single primary vortex, and hence is proportional to the half-width $b / 2$ of the microramp.

Using simple geometrical relations, the half-width can be expressed in terms of the half-span angle, ramp angle, and microramp height: 


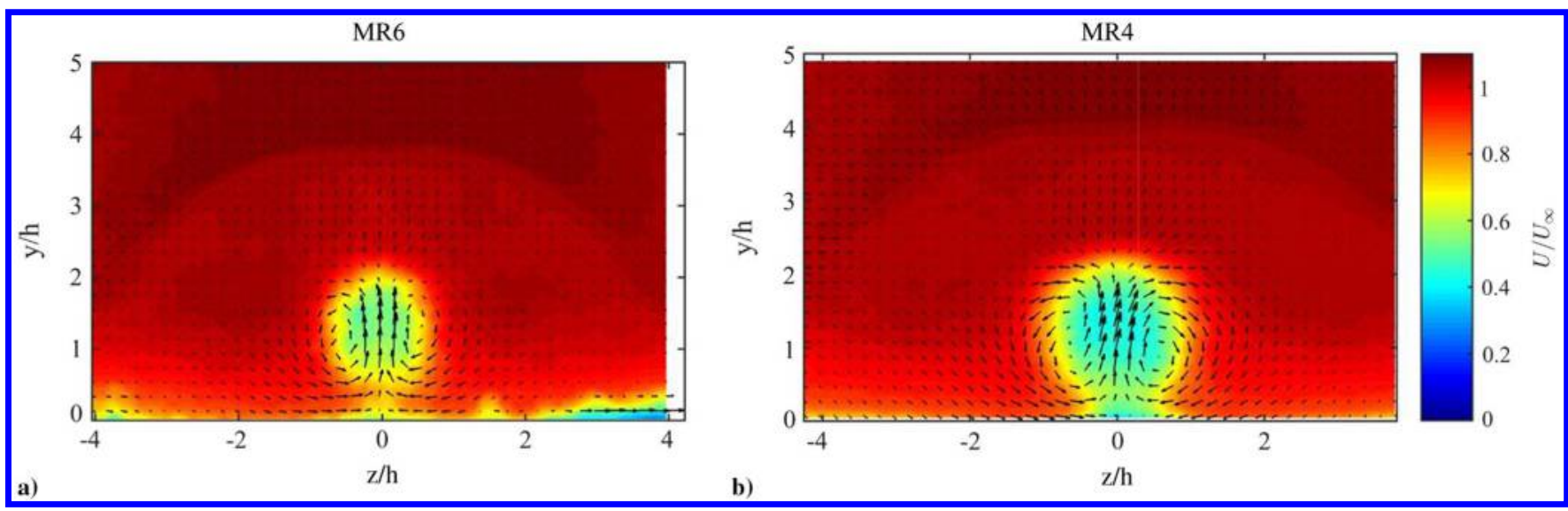

Fig. 10 Effect of low ramp angles on the crossflow field at $x / h=5$ with the contours of streamwise velocity: a) MR6 and b) MR4.

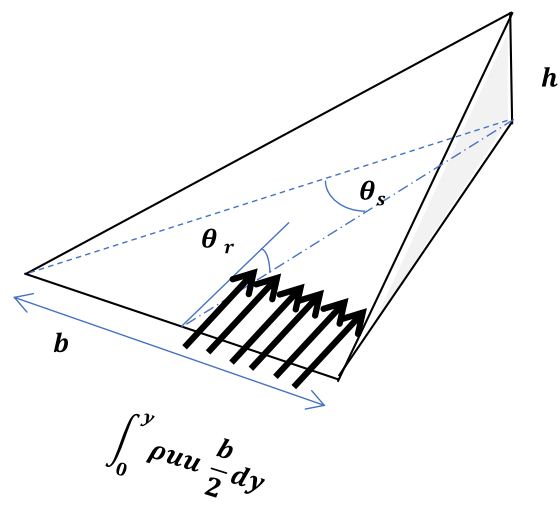

Fig. 11 Concept of captured momentum.

$$
\frac{b}{2}=h \frac{\tan \left(\theta_{s}\right)}{\tan \left(\theta_{r}\right)}
$$

and thus the captured momentum $E_{c}$ can be written as

$$
E_{c}=\frac{b}{2} \int_{0}^{y} \rho u u \mathrm{~d} y=h \frac{\tan \left(\theta_{s}\right)}{\tan \left(\theta_{r}\right)} \int_{0}^{y} \rho u u \mathrm{~d} y
$$

Here, the upper integration limit $y$ extends up to the part of the oncoming flow that enters the primary vortex. This extent is currently unknown, but it is expected to be governed by the device height $h$. In the present study, the device height is kept constant; therefore, the wall-normal extent of the captured momentum is expected to be the same for all the devices considered. Note that the factor $h \tan \left(\theta_{s}\right) / \tan \left(\theta_{r}\right)$ represents how the captured momentum changes with variations in the microramp geometry. However, it is also important to note that the second part in Eq. (2) depends on the incoming boundary-layer profile. Hence, this suggests that for similar upstream flow conditions, the half-width $\left[b / 2=h \tan \left(\theta_{s}\right) /\right.$ $\left.\tan \left(\theta_{r}\right)\right]$ can be used for the scaling of the various characteristics of the microramp wake when the geometrical configuration is varied.

\section{B. Circulation of the Primary Vortices}

The circulation of the primary vortices can be related to the geometrical parameters by a simplified conceptual analysis of the vortex formation. For this purpose, it is assumed that the stream tubes over the microramp surface leave the microramp edge without undergoing any spanwise turning. Thus, the streamwise velocity can be decomposed into a component normal and a component parallel to the microramp edge; see Fig. 12a. The edge-normal component $U \sin \left(\theta_{s}\right)$ has the most important contribution toward the formation of the primary vortex, whereas the parallel component $U \cos \left(\theta_{s}\right)$ does not contribute to the primary vortex directly. Figure $12 \mathrm{~b}$ shows that the edge-normal velocity component $U \sin \left(\theta_{s}\right)$ is being added as the tangential velocity component in the primary vortex over the length of the edge.

When viewed along the axis of the primary vortex, this would seem like there is a constant addition of tangential momentum at each radial location. Therefore, it can be assumed that for a larger length of the edge, a higher amount of tangential momentum is added to the vortex. Hence, the effective radius of the vortex at the trailing edge of the microramp can be assumed to be proportional to the length of the edge $C$ :

$$
R=K_{R} C=K_{R} \frac{h}{\cos \left(\theta_{s}\right) \tan \left(\theta_{r}\right)}\left(1+\frac{\left(\tan \left(\theta_{r}\right) \cos \left(\theta_{s}\right)\right)^{2}}{2}\right)
$$

Here, $K_{R}$ is a proportionality parameter, and the edge length $C$ has been expressed in terms of the geometrical parameters of the microramp. With this, the circulation at the end of the trailing edge can be written as

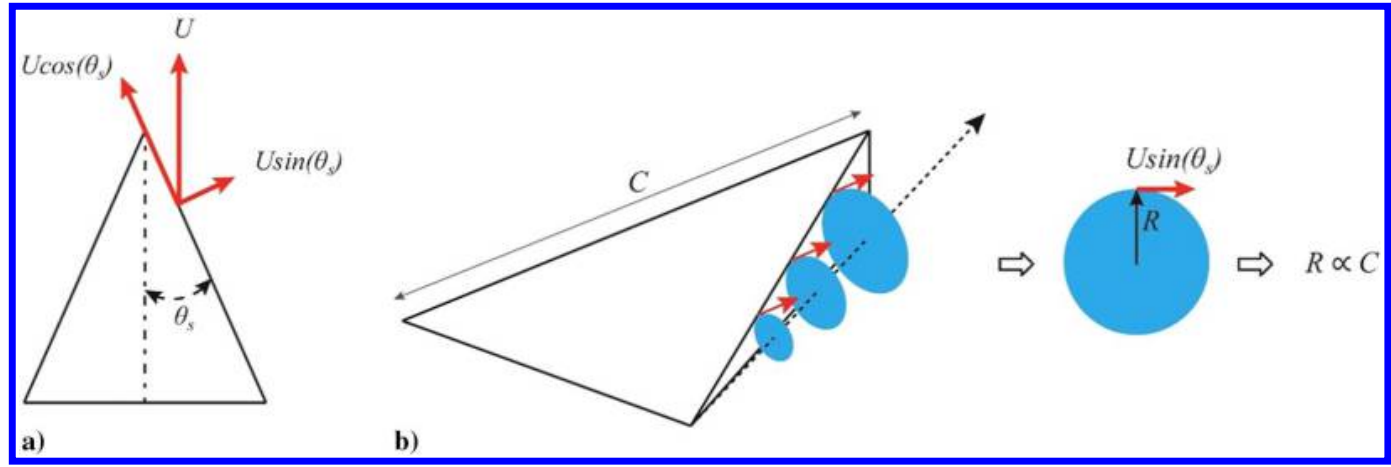

Fig. 12 Primary vortex formation: a) decomposition of the streamwise momentum in tangential and edgewise direction, and b) addition of the tangential momentum at various edgewise locations. 


$$
\Gamma_{\mathrm{TE}}=2 \pi U \sin \left(\theta_{s}\right) R=K_{R} 2 \pi U h \frac{\tan \left(\theta_{s}\right)}{\tan \left(\theta_{r}\right)}\left(1+\frac{\left(\tan \left(\theta_{r}\right) \cos \left(\theta_{s}\right)\right)^{2}}{2}\right)
$$

Equation (4) represents the circulation of a primary vortex at the trailing edge of the microramp. However, it is known from the literature $[5,16,18]$ that for a given microramp geometry, the circulation scales with its height and the friction velocity $u_{\tau}$. This scaling is therefore incorporated into Eq. (4), yielding

$$
\frac{\Gamma_{\mathrm{TE}}}{u_{\tau} h}=K_{R} \frac{U}{u_{\tau}} 2 \pi \frac{\tan \left(\theta_{s}\right)}{\tan \left(\theta_{r}\right)}\left(1+\frac{\left(\tan \left(\theta_{r}\right) \cos \left(\theta_{s}\right)\right)^{2}}{2}\right)
$$

The exact value of the oncoming streamwise velocity $U$ depends on the freestream conditions such that $U=K_{\mathrm{fs}} U_{\infty}$; here, the parameter $K_{\mathrm{fs}}$ accounts for the effect of the maximum wall-normal distance up to which the momentum is captured by the microramp. Therefore, $U / u_{\tau}=\left(K_{\mathrm{fs}} U_{\infty}\right) / u_{\tau}=K_{\mathrm{fs}} \sqrt{ }\left(2 / C_{f}\right)$. Here, $C_{f}=2\left(u_{\tau} / U_{\infty}\right)^{2}$ is the skin-friction coefficient. Incorporating this into Eq. (5),

$$
\frac{\Gamma_{\mathrm{TE}}}{u_{\tau} h}=K_{\mathrm{circ}} \sqrt{\frac{2}{C_{f}}} 2 \pi \frac{\tan \left(\theta_{s}\right)}{\tan \left(\theta_{r}\right)}\left(1+\frac{\left(\tan \left(\theta_{r}\right) \cos \left(\theta_{s}\right)\right)^{2}}{2}\right)
$$

Here, $K_{R} K_{\mathrm{fs}}$ is not directly known but is incorporated into the semiempirical parameter $K_{\text {circ }}=K_{R} K_{\mathrm{fs}}$.

In the present investigations $\left(20 \mathrm{deg}<\theta_{s}<45 \mathrm{deg}\right.$ and $4.3 \mathrm{deg}<$ $\left.\theta_{r}<21.3 \mathrm{deg}\right),\left(\tan \left(\theta_{r}\right) \cos \left(\theta_{s}\right)\right)^{2} / 2 \ll 1(0.01$ for baseline MR24 and maximum 0.06 for MR21). Therefore, Eq. (ㅁ) can be further simplified as

$$
\frac{\Gamma_{\mathrm{TE}}}{u_{\tau} h}=K_{\mathrm{circ}} \sqrt{\frac{2}{C_{f}}} 2 \pi \frac{\tan \left(\theta_{s}\right)}{\tan \left(\theta_{r}\right)}
$$

Since $h \tan \left(\theta_{s}\right) / \tan \left(\theta_{r}\right)=b / 2$, we can rewrite Eq. (7) as

$$
\frac{\Gamma_{\mathrm{TE}}}{u_{\tau} h}=K_{\mathrm{circ}} \sqrt{\frac{2}{C_{f}}} \pi \frac{b}{h}
$$

Comparing Eqs. (2) and (8) shows that at constant flow conditions upstream of the microramp and at constant device height, both circulation and captured momentum are proportional to the width $b$; therefore, their variation with the geometry is predicted to be similar.

Equation ( ) represents circulation just downstream of the microramp. It is known that the circulation decreases downstream of the microramp [17]. This downstream evolution of circulation depends on the oncoming flow and the streamwise location $(x / h)$. This effect is incorporated by adding a term $K_{1}$, which is an empirical parameter that varies with the streamwise location $(x / h)$ :

$$
\frac{\Gamma_{x / h}}{u_{\tau} h}=\sqrt{\frac{2}{C_{f}}}\left(K_{\mathrm{circ}} \pi \frac{b}{h}+K_{1}\right)
$$

It should be noted that both model parameters $K_{\text {circ }}$ and $K_{1}$ depend on the upstream flow conditions and boundary-layer properties, and therefore need empirical calibration.

\section{Model Validation}

\section{A. Circulation of the Primary Vortex Pair}

Figure 13 shows the time-averaged vorticity contours obtained from stereographic PIV in the crossflow plane located at $x / h=5$. The circulation of each vortex in the pair is calculated by the area integration of the $x$ vorticity $\omega_{x}$ separately. The mean value of individual circulation magnitudes of the two vortices (absolute value) is considered as the circulation of the vortex pair.

Figure 14a shows the experimental values of circulation measured for the ramp angle sweep at $x / h=5$. To calibrate the model, Eq. (9) is

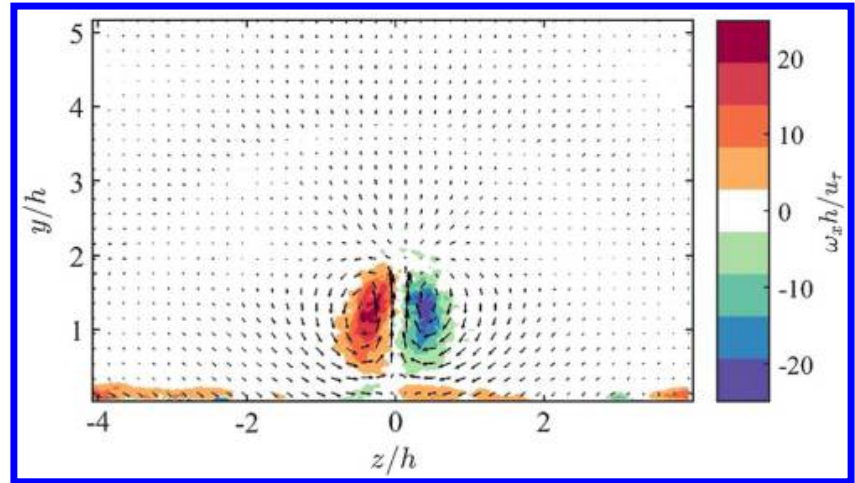

Fig. 13 Vorticity contours in the crossflow plane at $x / h=5$ (MR24).

fitted to these circulation values in the ramp sweep $\left(\theta_{r}=6.45\right.$ to 21.3 deg see Fig. 14a); and values of parameters $K_{\text {circ }}=0.0264$ and $K_{1}=0.1514$ are determined. For the smallest ramp angle of $\theta_{r}=$ $4.3 \mathrm{deg}$ (MR4), the circulation is observed to be lower than expected due to the increased interaction between the vortex and the wall; see Fig. 10b. Since such effect is not taken into account while formulating the model, the case of $\theta_{r}=4.3 \mathrm{deg}$ is not used to calibrate the model. This calibrated equation [Eq. ()] is subsequently used to predict the circulation values for the half-span angle sweep. As shown in Fig. 14b, the model prediction is in a good agreement with the experimental measurements for $\theta_{s}=20$ to $28 \mathrm{deg}$. The trends of the model are consistent with the assumption that the circulation strength is proportional to the captured momentum, and hence the microramp width. Therefore, when increasing the ramp angle at a constant half-span angle, the microramp width and the circulation will decrease (Fig. 14a); whereas increasing the half-span angle at a constant ramp angle will increase the microramp width and circulation (Fig. 14b).

The model overpredicts the circulation for the larger half-span angles because the model does not account for the delayed mutual interaction (as shown in Figs. 7-9) and increased vortex-wall interaction. For the higher half-span angles, the vortex core leaves the microramp edge earlier (see Fig. 8). As a result, a lower amount of captured momentum goes into the vortex core. Also, a larger separation between the two vortex cores delays the formation of the mutual upwash further downstream. Due to a weaker upwash just downstream of the trailing edge, the cores stay closer to the wall where viscous effects may lead to lower values of circulation. Comparing crossflow fields (Figs. 4a, 9a, and 9b) with the predicted circulation (Fig. 14b) shows that the model predictions are better when the primary vortices are sufficiently far away from the wall such that the streamwise momentum deficit region is isolated from the wall. A similar argument can be made for the case of low ramp angles; e.g., comparing Figs. 10b and 10a shows increased vortex interaction with the wall for lower ramp angle MR4 compared to MR6. This also leads to the momentum deficit region extending toward the wall for MR4; here, the circulation of MR4 does not follow the model trend as compared to MR6; see Fig. 14a.

Equation (8) suggests that the half-width of the microramp, $b / 2=$ $h \tan \left(\theta_{s}\right) / \tan \left(\theta_{r}\right)$, has a significant influence on the primary vortex circulation. To further investigate this relation, the circulation is plotted against the half-width in Fig. 15. It can be observed that the values of circulation are similar for the microramps with similar halfwidths, even for different combinations of sweep and ramp angle. This can be seen as a confirmation that the vortex strength directly depends on the amount of captured momentum and how this momentum is distributed to the vortex core. Deviations from this trend are again observed for the higher half-span angles and low ramp angle. Moreover, Fig. 15 suggests that there exist configurations, other than the baseline MR24, that can maximize the circulation.

\section{B. Wake Velocity}

The streamwise evolution of the wake velocity, which is the minimum streamwise velocity in the wake, is a convenient parameter to assess the effectiveness of the primary vortices in filling up the wake. 


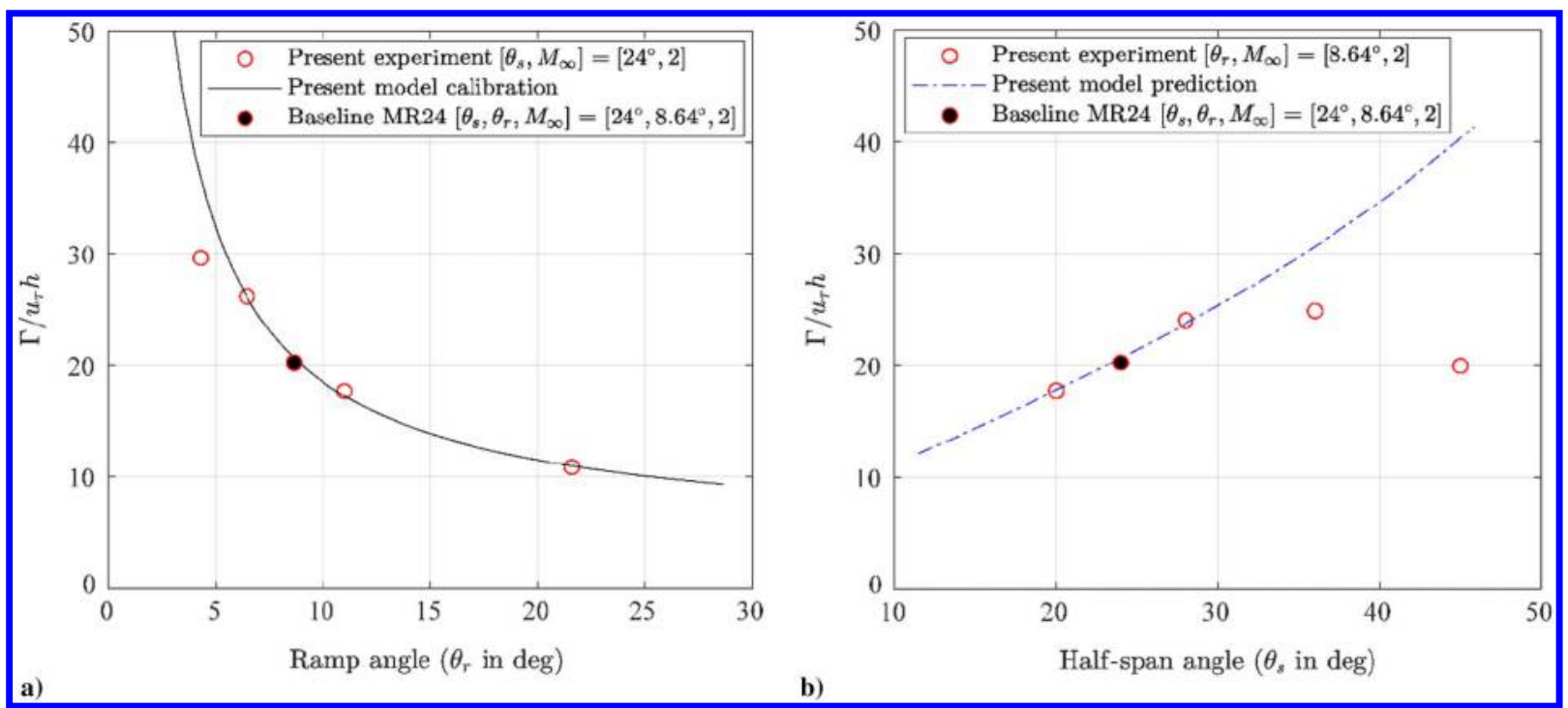

Fig. 14 Circulation of the primary vortices at $x / h=5$ for all the configurations, including a) model calibration and b) prediction.

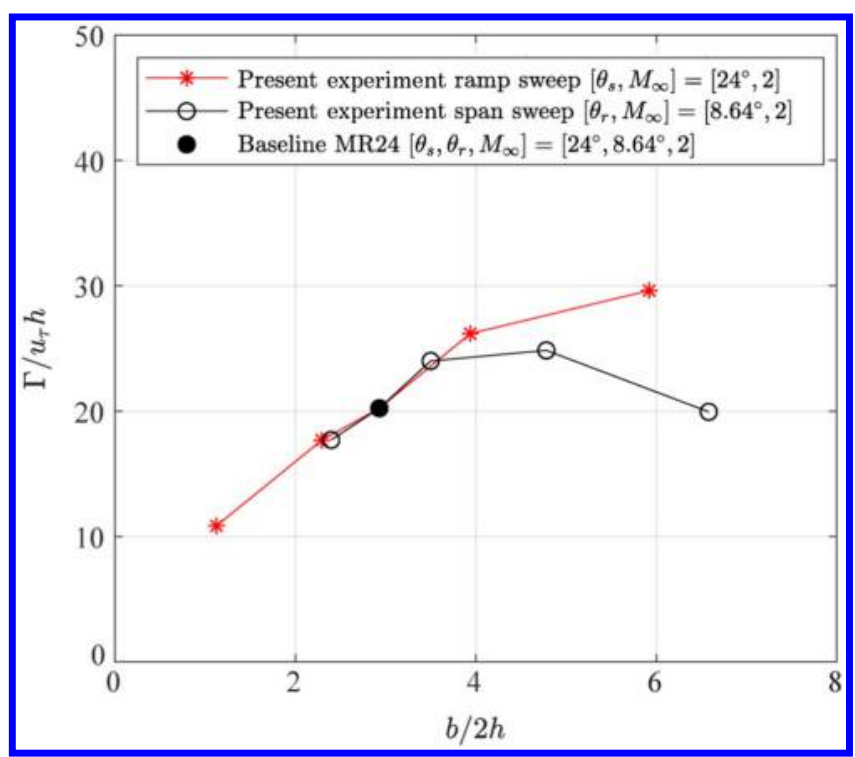

Fig. 15 Effect of half-width on the normalized circulation of primary vortices.
As shown in Fig. 16, it is observed that for every configuration, the wake velocity is low at first and gradually increases with the streamwise distance. The nondimensional wake velocity approaches values ranging from 0.35 to 0.85 within the measurement domain, depending on the geometry.

In various studies, the microramp height is used as the length scale to nondimensionalize the wake properties. Giepman et al. [12] tested the Anderson microramp with different device heights and found that the graphs of streamwise evolution of the wake velocity appear to collapse when the streamwise coordinate is scaled with the ramp height.

In the present study, the height is kept constant; and still a significant difference in the wake velocity evolution is observed for different half-span and ramp angles (see Fig. 16). This suggests that apart from the microramp height, other parameters have an effect on the wake velocity evolution as well.

Since it was postulated in Sec. V. A that the wake behavior strongly depends on the captured momentum [Eq. (2)], the geometry term $h \tan \left(\theta_{s}\right) / \tan \left(\theta_{r}\right)$ is used as a scaling factor instead of $h$. The results for the wake velocity using the conventional scaling in Fig. 16 all appear to collapse around a common curve when scaled with the new scaling factor; see Fig. 17. This provides an additional indication that the captured momentum concept indeed makes sense when
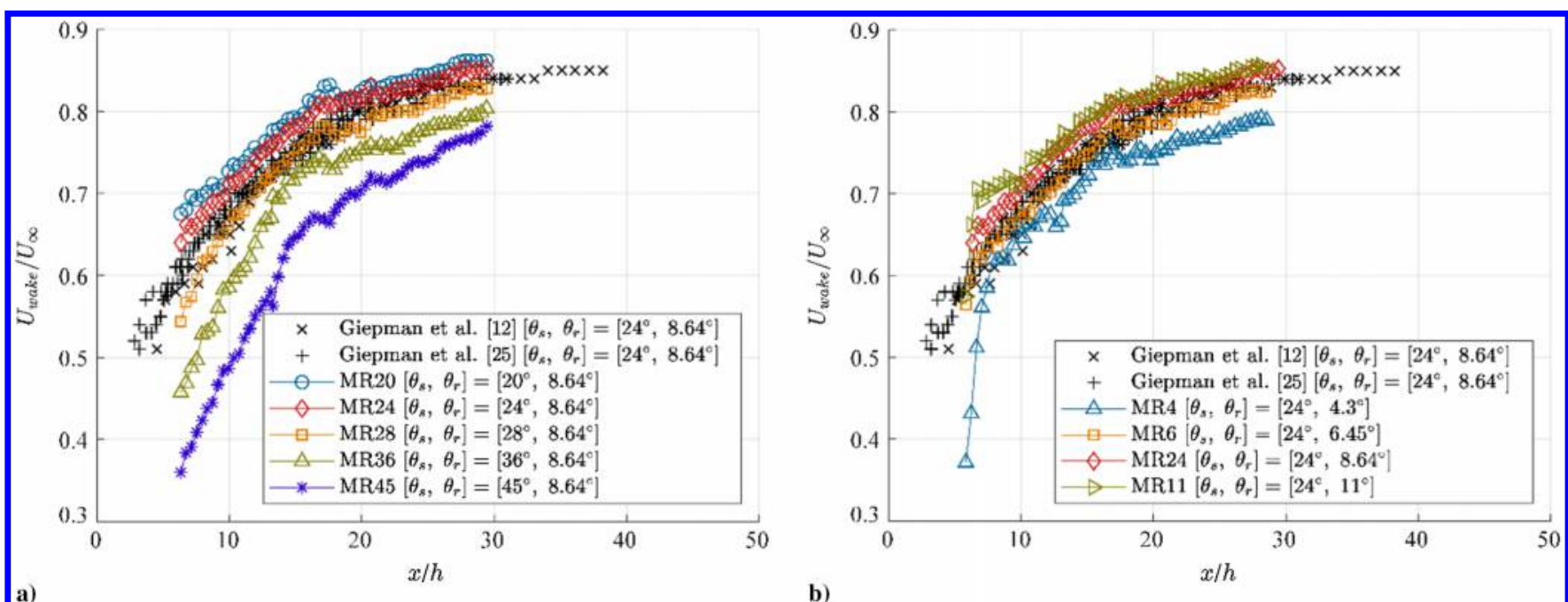

Fig. 16 Streamwise evolution of wake velocity for a) half-span angle sweep and b) ramp angle sweep, including data from literature [12,25]. 


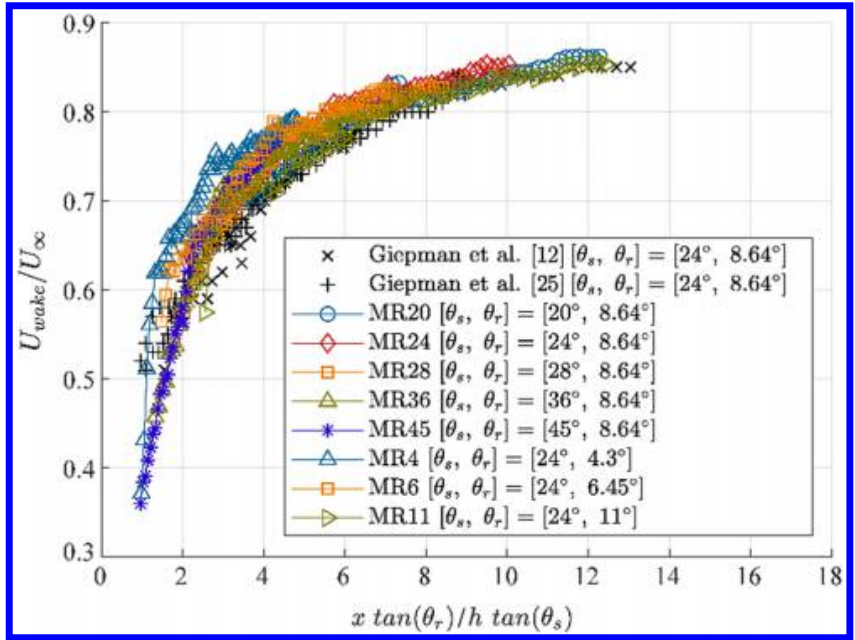

Fig. 17 Streamwise evolution of wake velocity with the present scaling, all configurations, including data from literature $[\underline{12,25}]$.

describing the wake dynamics. However, the exact mechanism of this dependence is still to be explored.

\section{Wake Location}

The evolution of the wake location is affected by changing the halfspan angle, but it does not significantly depend on the ramp angle (see Fig. 18). Note that when the ramp angle is changed, the span, captured momentum, and edge are changing as well (see Table 2), but they do not affect the wall-normal location of the wake. Observing Figs. 6a and $6 \mathrm{~b}$ reveals a similar trend, where the wall-normal location of the maximum upwash is significantly affected by the half-span angle but not by the ramp angle. Furthermore, Figs. $7 \mathrm{a}$ and $7 \mathrm{~b}$ show that just downstream of a microramp around $x / h=\overline{3}$, the separation between the vortex cores increases more with the increasing half-span angle than with the increasing ramp angle. When the separation between the two primary vortex cores for a given strength is larger, the wake remains closer to the wall. This effect is negligible with the changing ramp angle.

Figure 19 shows the wake location evolution scaled with the scale factor $h \tan \left(\theta_{s}\right) / \tan \left(\theta_{r}\right)$. Since the ramp angle does not affect the wake location, the corresponding curves do not follow the new scaling. Figure 20 shows that the curves of wake location appear to overlap when the term $\tan \left(\theta_{r}\right)$ is dropped from the scaling factor, and the streamwise evolution is scaled using $h \tan \left(\theta_{s}\right)$. The present measurements agree with the measurements of Giepman et al. [12].

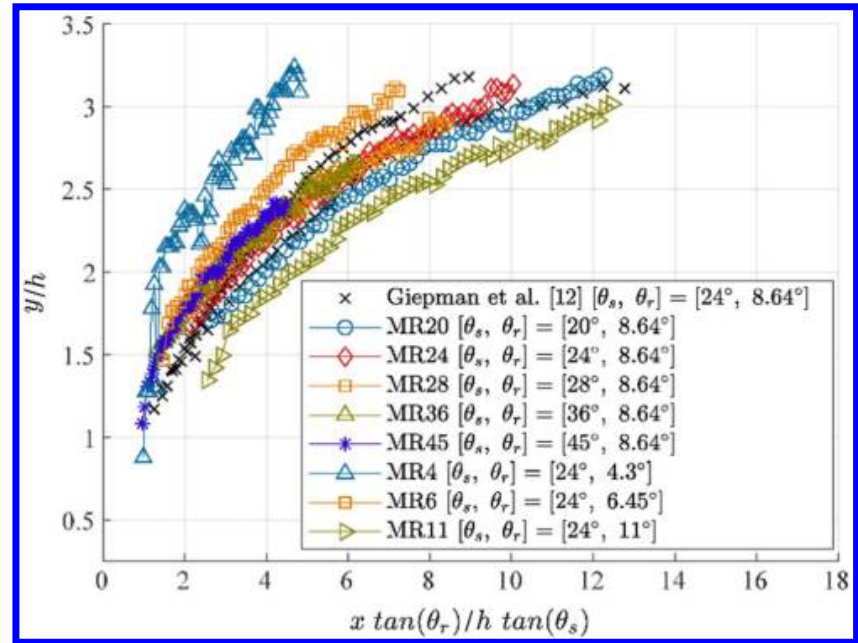

Fig. 19 Streamwise evolution of wake location scaled with the present scaling factor, including the data from literature [12].

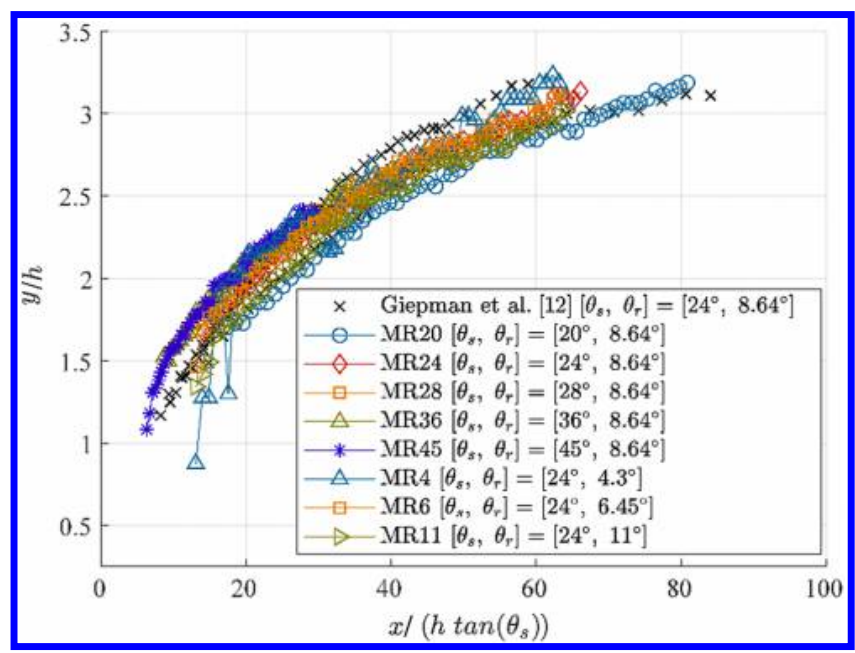

Fig. 20 Streamwise evolution of wake location scaled with $h \tan \left(\theta_{s}\right)$, including the data from literature [12].

\section{Incompressible Added Momentum}

The effectiveness of the microramp in its flow control authority can be judged based on the amount of momentum added in the near-wall region. Giepman et al. [12] found that the shock-induced separation bubble is sensitive mainly to the boundary-layer momentum below
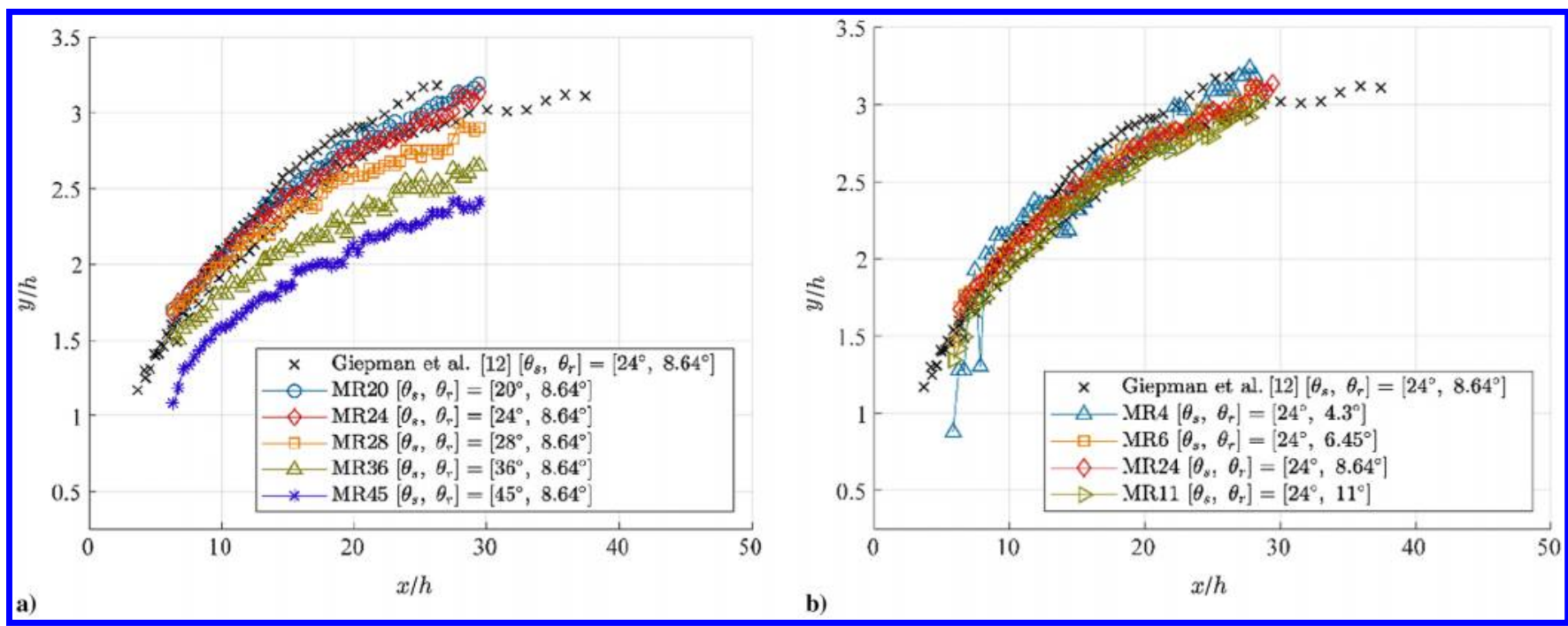

Fig. 18 Streamwise evolution of wake location: a) half-span angle sweep and b) ramp angle sweep, including data from literature [12]. 
$0.43 \delta_{99}$. Therefore, to quantify the effectiveness of a microramp, the concept of (incompressible) added momentum in the symmetry plane of the microramp was introduced [12]:

$$
E_{\text {added }}=\int_{0}^{0.43 \delta_{99}} \frac{U^{2}-U_{\text {clean }}^{2}}{U_{\infty}^{2}} \mathrm{~d} y
$$

Here, $U_{\text {clean }}$ corresponds to the velocity profile of the undisturbed boundary layer. The streamwise evolution of the incompressible added momentum for different configurations is shown in the Fig. 21. It is observed that up to around $x / h=17$, momentum is being added to the boundary layer. Thereafter, the added momentum reaches a plateau. This trend is consistent with the measurements of Giepman et al. [12]. It is observed that for the higher ramp angles and lower span angles, the added momentum in the symmetry plane is overall higher.

In the case of the ramp angle sweep, the streamwise evolution of the incompressible added momentum properly scales with the present scaling; however, it does not do so in the case of the half-span angle sweep (see Fig. 22). This can again be linked to the larger vortex core separation for larger half-span angles (see Fig. 8). In the case of the half-span sweep, with an increased separation, the vortex cores stay closer to the wall (see Fig. 18a). When the vortex core is away from the symmetry plane and closer to the wall, its effectiveness in bringing high-momentum fluid from the surrounding flow toward the symmetry plane reduces. This results in the lower value of the added incompressible momentum. In the case of the ramp angle sweep, the core separation increases with decreasing ramp angle. But, the location of the vortex core is also a bit higher (see Fig. 18b). This results in a more effective addition of momentum in the symmetry plane.

\section{Conclusions}

The physical relation between microramp geometry and the downstream flow characteristics has been investigated by means of an experimental parametric study. The microramp geometry is parameterized using three parameters: height $h$, half-span angle $\theta_{s}$, and ramp angle $\theta_{r}$. The different test configurations are derived by varying the latter two while keeping the device height constant throughout the study.

For the same device height and incoming boundary-layer flow, changes in the half-span angle or ramp angle are found to have a significant influence on the flow characteristics. Increasing the halfspan angle or decreasing the ramp angle has similar effects on the flow characteristics of the microramp wake. These effects include decreased
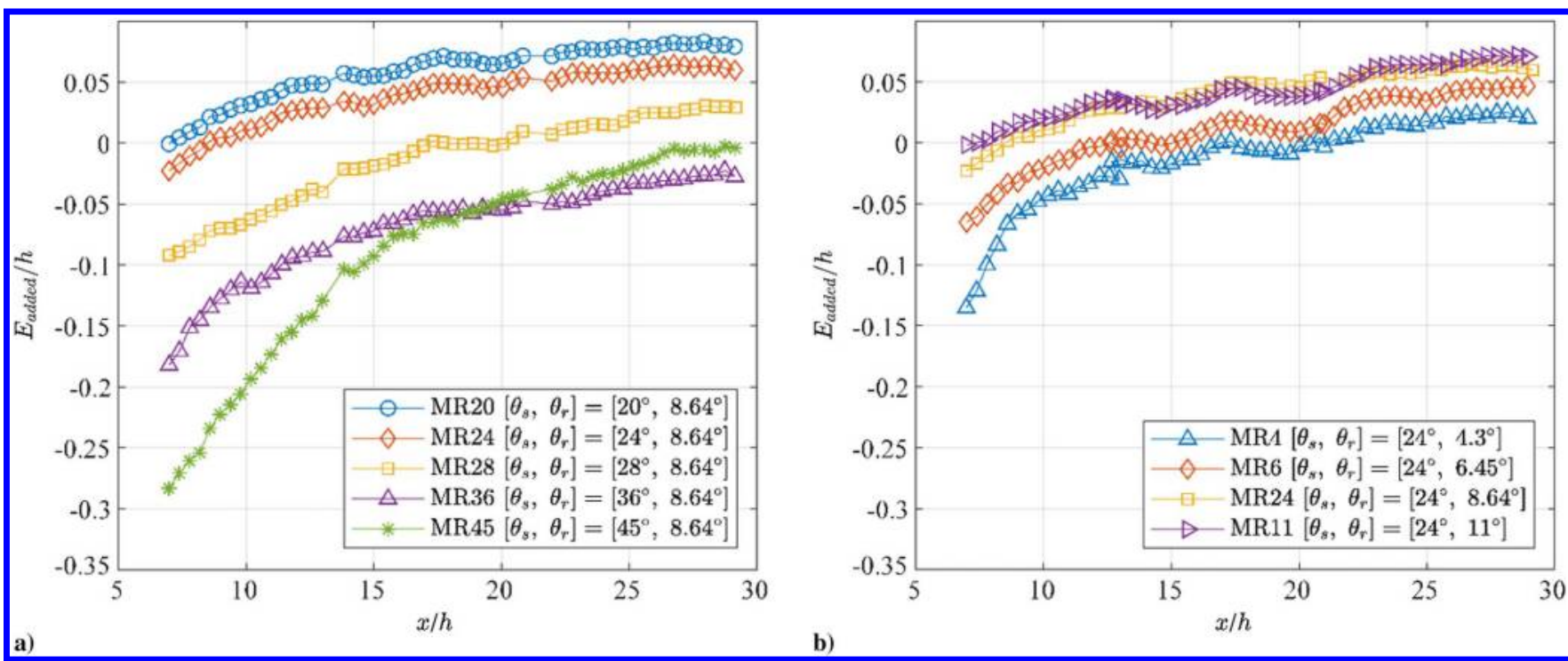

Fig. 21 Streamwise evolution of incompressible added momentum measured in the symmetry plane: a) half-span angle sweep and b) ramp angle sweep.
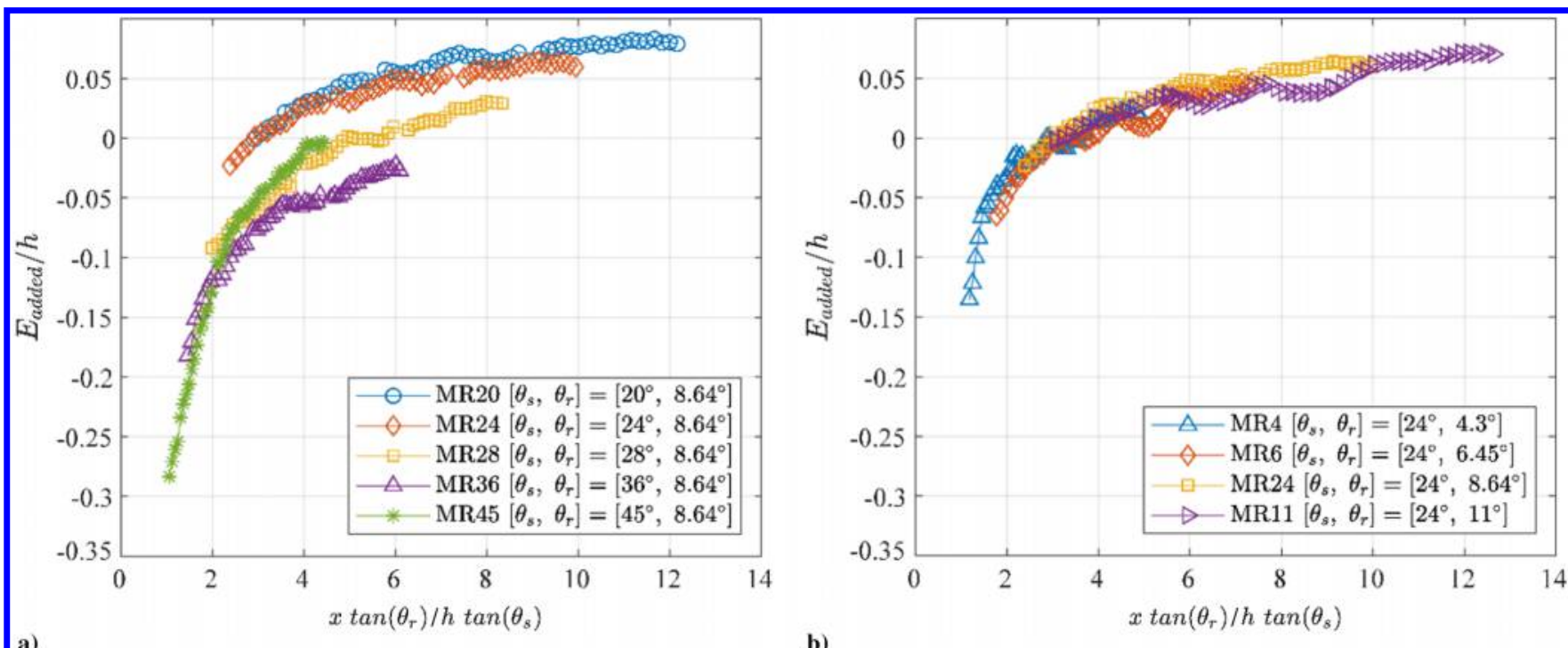

Fig. 22 Streamwise evolution of the added incompressible momentum with present scaling: a) half-span angle sweep and b) ramp angle sweep. 
wake velocity, larger vortex core separation, and a lower amount of (incompressible) added momentum. However, the mutual upwash induced by the two primary vortices is more sensitive to the ramp angle than to the half-span angle. It increases with the lowering of the ramp angle but remains nearly the same with changes in the half-span angle. With an increasing half-span angle, the vortex core leaves the microramp at a lower edge length; this behavior is not observed when the ramp angle is changed.

To provide a physical reasoning to the observed effects, a physical model has been constructed. This model includes the concept of captured momentum as a mechanism by which this generates the primary (streamwise) vortex structures. As such, it relates the microramp geometry to the vortex circulation and the geometrical scaling of the flowfield. Since the microramp is a passive device, its working mechanism to generate streamwise vorticity largely depends on redistributing the momentum in the incoming flow. The momentum that faces the microramp edge is partly transferred into the primary vortices. This momentum is referred to here as the captured momentum, and it governs the flow characteristics of the microramp wake. For similar incoming flow conditions, the captured momentum, corresponding to one primary vortex, is directly proportional to the half-width of the microramp. Hence, the half-width is used as a length scale for scaling of different flow characteristics of the microramp wake. It is found that to a satisfactory extent, the plots of wake velocity evolution appear to collapse around a common curve when the streamwise coordinate is scaled with the half-width. A similar scaling effect is observed in the added incompressible momentum for the cases in ramp angle sweep. In the case of the half-span angle sweep, the scaled plots shift downward with the increasing half-span angle, suggesting the decreasing effectiveness in momentum addition in the symmetry plane. This is linked to the increased separation of the primary vortex cores as the span angle increases. The wall-normal wake location scales with the half-width only in the case of the half-span angle sweep, but it does not depend strongly on the ramp angle.

Based on the conceptual model, a simplified relation between primary vortex circulation and geometry parameters is formulated in this study. This relation still contains empirical parameters, but once these are determined for the flow conditions under consideration, a valid correlation is provided for half-span angles within $\theta_{s}=$ 20 to $28 \mathrm{deg}$ for the ramp angles within $\theta_{r}=6.45$ to $21.3 \mathrm{deg}$.

The presented relation between the microramp geometry and the wake characteristics can be useful in designing an effective flow control, tailored for a specific application. For example, the ramp and half-span angles can be adjusted to achieve a desired primary vortex strength along with the desired wall-normal wake location such that the microramp wake causes minimum disturbance to the outer flow. Moreover, the scaled wake evolution presented here can be used to achieve a specific wake characteristic at a prescribed physical location. This could be useful in applications involving size restrictions, e.g., intakes, compressor blades, etc. Finally, the presented model for the primary vortex circulation and the scaled wake evolution can be used for a preliminary optimization of microramps to achieve desired wake characteristic within the constraints of the specific application.

\section{References}

[1] Galbraith, M. C., Orkwis, P. D., and Benek, J. A., "Multi-Row MicroRamp Actuators for Shock Wave Boundary-Layer Interaction Control," 47th AIAA Aerospace Sciences Meeting Including the New Horizons Forum and Aerospace Exposition, AIAA Paper 2009-0321, Jan. 2009, pp. $1-30$. https://doi.org/10.2514/6.2009-321

-[2] Panaras, A. G., and Lu, F. K., "Micro-Vortex Generators for Shock Wave/Boundary Layer Interactions," Progress in Aerospace Sciences, Vol. 74, April 2015, pp. 16-47. https://doi.org/10.1016/j.paerosci.2014.12.006

-[3] Delery, J. M., "Shock Wave/Turbulent Boundary Layer Interaction and Its Control," Progress in Aerospace Sciences, Vol. 22, No. 4, 1985, pp. 209-280. https://doi.org/10.1016/0376-0421(85)90001-6

[4] Giepman, R. H. M., Louman, R., Schrijer, F. F. J., and van Oudheusden, B. W., "Experimental Investigation of Boundary Layer Tripping Devices for Shock Wave-Boundary Layer Control," 45th AIAA Fluid
Dynamics Conference, AIAA Paper 2015-2780, June 2015, pp. 1-18. https://doi.org/10.2514/6.2015-2780

[5] Giepman, R., "Flow Control for Oblique Shock Wave Reflections," $\mathrm{Ph} . D$. Dissertation, Delft Univ. of Technology, Delft, The Netherlands, 2016.

https://doi.org/10.4233/uuid:597489bb-0b41-4da2-8ec4-b1bc390f8d2c

- [6] Zhang, B., Zhao, Q., Xiang, X., and Xu, J., "An Improved Micro-Vortex Generator in Supersonic Flows," Aerospace Science and Technology, Vol. 47, Dec. 2015, pp. 210-215. https://doi.org/10.1016/j.ast.2015.09.029

[7] Lin, J. C., "Review of Research on Low-Profile Vortex Generators to Control Boundary-Layer Separation," Progress in Aerospace Sciences, Vol. 38, Nos. 4-5, 2002, pp. 389-420. https://doi.org/10.1016/S0376-0421(02)00010-6

[8] Lu, F. K., Li, Q., and Liu, C., "Microvortex Generators in HighSpeed Flow," Progress in Aerospace Sciences, Vol. 53, Aug. 2012, pp. 30-45. https://doi.org/10.1016/j.paerosci.2012.03.003

- [9] Anderson, B., Tinapple, J., and Surber, L., "Optimal Control of Shock Wave Turbulent Boundary Layer Interactions Using Micro-Array Actuation," 3rd AIAA Flow Control Conference, AIAA Paper 2006-3197, 2006, pp. 1-14. https://doi.org/10.2514/6.2006-3197

[10] Berrier, B., and Allan, B., "Experimental and Computational Evaluation of Flush-Mounted, S-Duct Inlets," 42nd AIAA Aerospace Sciences Meeting and Exhibit, AIAA Paper 2004-0764, 2004, pp. 1-15. https://doi.org/10.2514/6.2004-764

[11] Blinde, P. L., Humble, R. A., van Oudheusden, B. W., and Scarano, F., "Effects of Micro-Ramps on a Shock Wave/Turbulent Boundary Layer Interaction," Shock Waves, Vol. 19, No. 6, 2009, pp. 507-520. https://doi.org/10.1007/s00193-009-0231-9

[12] Giepman, R. H. M., Schrijer, F. F. J., and Van Oudheusden, B. W., "Flow Control of an Oblique Shock Wave Reflection with Micro-Ramp Vortex Generators: Effects of Location and Size," Physics of Fluids, Vol. 26, No. 6, 2014, Paper 066101. https://doi.org/10.1063/1.4881941

[13] Qing-Hu, Z., Shi-He, Y., Yang-Zhu, Z., Zhi, C., and Yu, W., "The Effect of Micro-Ramps on Supersonic Flow over a Forward-Facing Step," Chinese Physics Letters, Vol. 30, No. 4, 2013, Paper 044701. https://doi.org/10.1088/0256-307X/30/4/044701

[14] Funderburk, M. L., and Narayanaswamy, V., "Experimental Investigation of Microramp Control of an Axisymmetric Shock/Boundary-Layer Interaction," AIAA Journal, Vol. 57, No. 8, 2019, pp. 3379-3394. https://doi.org/10.2514/1.J057846

[15] Ashill, P. R., Fulker, J. L., and Hackett, K. C., "Studies of Flows Induced by Sub-Boundary Layer Vortex Generators(SBVGs)," AIAA Paper 2002-0968, 2002.

[16] Nolan, W. R., and Babinsky, H., "Characterization of Micro-Vortex Generators in Supersonic Flows," 49th AIAA Aerospace Sciences Meeting Including the New Horizons Forum and Aerospace Exposition, AIAA Paper 2011-0071, 2011.

[17] Nolan, W. R., and Babinsky, H., "Comparison of Micro-Vortex Generators in Supersonic Flows," 6th AIAA Flow Control Conference, AIAA Paper 2012-2812, 2012, pp. 1-13. https://doi.org/10.2514/6.2012-2812

[18] Sun, Z., "Micro Ramps in Supersonic Turbulent Boundary Layers: An Experimental and Numerical Study," Ph.D. Thesis, Faculty of Aerospace Engineering, Delft Univ. of Technology, Delft, The Netherlands, 2014.

[19] Wang, X., Yan, Y., Sun, Z., and Liu, C., "The Vortical Structures in the Rear Separation and Wake Produced by a Supersonic Micro-Ramp," Flow, Turbulence and Combustion, Vol. 93, No. 1, 2014, pp. 25-36. https://doi.org/10.1007/s10494-014-9531-y

[20] Li, Q., and Liu, C., "Implicit LES for Supersonic Microramp Vortex Generator: New Discoveries and New Mechanisms," Modelling and Simulation in Engineering, Vol. 2011, April 2011, Paper 934982. https://doi.org/10.1155/2011/934982

[21] Lu, F. K., Pierce, A. J., and Shih, Y., "Experimental Study of near Wake of Micro Vortex Generators in Supersonic Flow," AIAA Paper 2010-4623, 2010. https://doi.org/10.2514/6.2010-4623

[22] Li, Q., and Liu, C., "Declining Angle Effects of the Trailing Edge of a Microramp Vortex Generator," Journal of Aircraft, Vol. 47, No. 6, 2010, pp. 2086-2095. https://doi.org/10.2514/1.C000318

[23] Lu, F. K., Pierce, A. J., Shih, Y., Liu, C., and Li, Q., "Experimental and Numerical Study of Flow Topology Past Micro Vortex Generators," AIAA 40th Fluid Dynamics Conference and Exhibit, AIAA Paper 20104463, 2010, pp. 1-13. 
[24] Lee, S., and Loth, E., "Supersonic Boundary-Layer Interactions with Various Micro-Vortex Generator Geometries," AIAA Paper 2009-3712, 2009.

https://doi.org/10.1017/S0001924000003353

[25] Giepman, R. H. M., Srivastava, A., Schrijer, F. F. J., and van Oudheusden, B. W., "The Effects of Mach and Reynolds Number on the Flow Mixing Properties of Micro-Ramp Vortex Generators in a Supersonic Boundary Layers," 45th AIAA Fluid Dynamics Conference, AIAA Paper 2015-2779, 2015.

[26] Babinsky, H., Li, Y., and Ford, C. W. P., "Microramp Control of Supersonic Oblique Shock-Wave/Boundary-Layer Interactions," AIAA Journal, Vol. 47, No. 3, 2009, pp. 668-675. https://doi.org/10.2514/1.38022

[27] Lee, S., Loth, E., and Babinsky, H., "Normal Shock Boundary Layer Control with Various Vortex Generator Geometries," Computers and Fluids, Vol. 49, No. 1, 2011, pp. 233-246. https://doi.org/10.1016/j.compfluid.2011.06.003

[28] Li, L.-Q., Huang, W., Yan, L., Du, Z.- B., and Fang, M., "Numerical Investigation and Optimization on the Micro-Ramp Vortex Generator Within Scramjet Combustors with the Transverse Hydrogen Jet," Aerospace Science and Technology, Vol. 84, Jan. 2019, pp. 570-584. https://doi.org/10.1016/j.ast.2018.11.011

[29] Clauser, F. H., "The Turbulent Boundary Layer," Advances in Applied Mechanics, edited by H. L. Dryden, and T. von Kármán, Elsevier, New York, 1956, pp. 1-51.
[30] Van Driest, E. R., "On Turbulent Flow near a Wall," Journal of the Aeronautical Sciences, Vol. 23, No. 11, 1956, pp. 1007-1011. https://doi.org/10.2514/8.3713

[31] White, F. M., Viscous Fluid Flow, MacGraw-Hill Education, New York, 2011.

[32] Kahler, C., Sammler, B., and Kompenhans, J., "Generation and Control of Tracer Particles for Optical Flow Investigations in Air," Experiments in Fluids, Vol. 33, No. 6, 2002, pp. 736-742. https://doi.org/10.1007/s00348-002-0492-x

[33] Ragni, D., Schrijer, F., Van Oudheusden, B. W., and Scarano, F., "Particle Tracer Response Across Shocks Measured by PIV," Experiments in Fluids, Vol. 50, No. 1, 2011, pp. 53-64. https://doi.org/10.1007/s00348-010-0892-2

[34] Benedict, L. H., and Gould, R. D., "Towards Better Uncertainty Estimates for Turbulence Statistics," Experiments in Fluids, Vol. 22, No. 2, 1996, pp. 129-136. https://doi.org/10.1007/s003480050030

[35] Schrijer, F. F. J., and Scarano, F., "Effect of Predictor-Corrector Filtering on the Stability and Spatial Resolution of Iterative PIV Interrogation," Experiments in Fluids, Vol. 45, No. 5, 2008, pp. $927-$ 941.

https://doi.org/10.1007/s00348-008-0511-7

P. Lavoie Associate Editor 